CHERCHER, REPÉRER, AVANCER

\title{
DERRIÈRE LA PLAGE, LES PLANTATIONS
}

Touristification du littoral et recomposition des élites dans le Nordeste brésilien Tristan Loloum

\section{Le Seuil | «Actes de la recherche en sciences sociales »}

$2017 / 3 \mathrm{~N}^{\circ} 218$ | pages 46 à 63

ISSN 0335-5322

ISBN 9782021340587

Article disponible en ligne à l'adresse :

http://www.cairn.info/revue-actes-de-la-recherche-en-sciencessociales-2017-3-page-46.htm

\section{Pour citer cet article :}

Tristan Loloum, « Derrière la plage, les plantations. Touristification du littoral et recomposition des élites dans le Nordeste brésilien », Actes de la recherche en sciences sociales 2017/3 ( $\left.\mathrm{N}^{\circ} 218\right)$, p. 46-63.

DOI 10.3917/arss.218.0046

Distribution électronique Cairn.info pour Le Seuil.

(C) Le Seuil. Tous droits réservés pour tous pays.

La reproduction ou représentation de cet article, notamment par photocopie, n'est autorisée que dans les limites des conditions générales d'utilisation du site ou, le cas échéant, des conditions générales de la licence souscrite par votre établissement. Toute autre reproduction ou représentation, en tout ou partie, sous quelque forme et de quelque manière que ce soit, est interdite sauf accord préalable et écrit de l'éditeur, en dehors des cas prévus par la législation en vigueur en France. Il est précisé que son stockage dans une base de données est également interdit. 

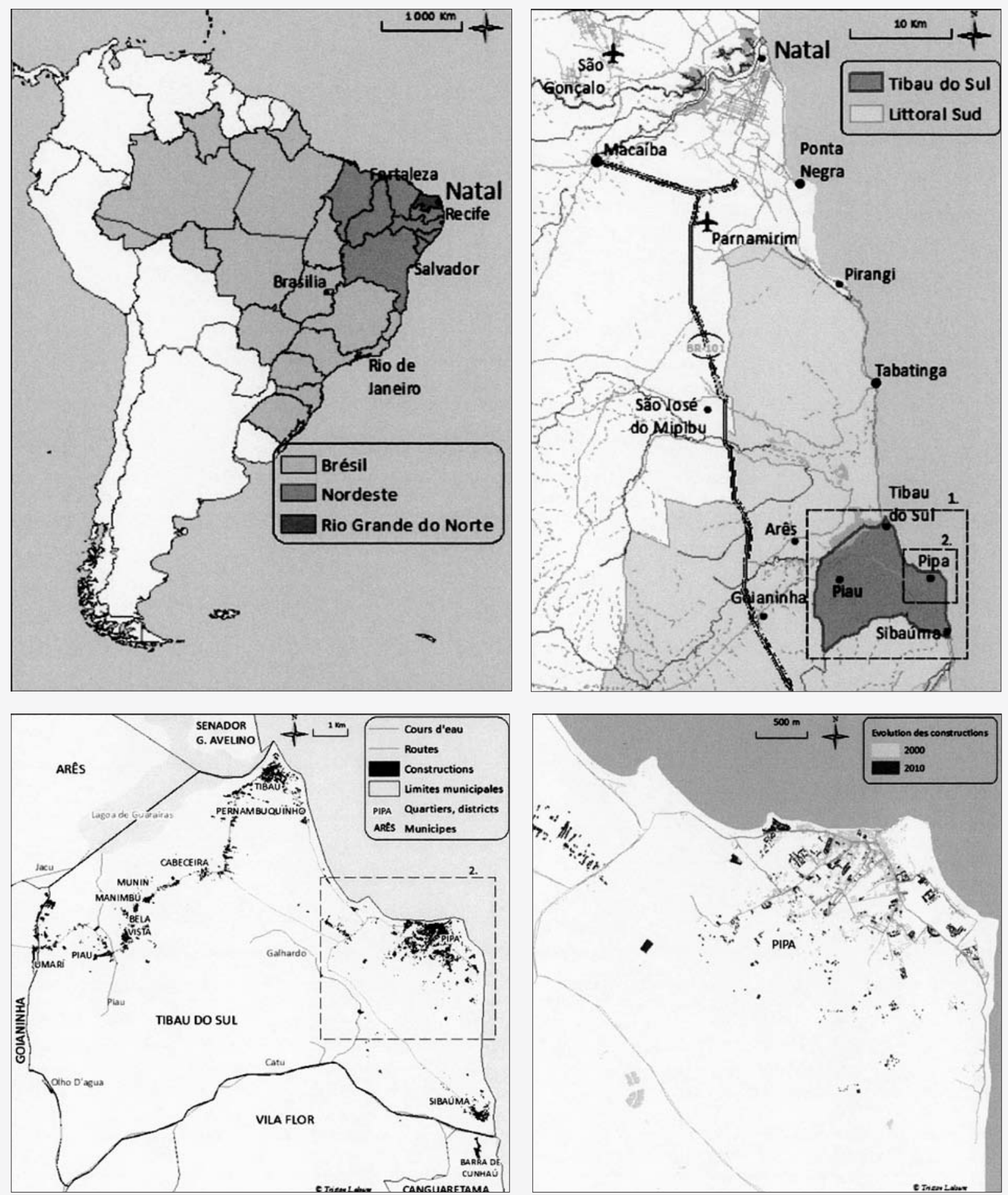

ILLUSTRATION 1. Praia da Pipa est la deuxième destination touristique de l'Etat du Rio Grande do Norte, après Natal, sa capitale. 


\title{
Tristan Loloum
}

\section{Derrière la plage, les plantations}

\author{
Touristification du littoral et recomposition des élites dans le Nordeste brésilien
}

L'invention du tourisme balnéaire a longtemps été appréhendée comme le produit d'un « désir de rivage » porté par les catégories sociales dominantes, qu'il s'agisse des médecins hygiénistes (prescripteurs des «bains de mer » à visée thérapeutique), des artistes et écrivains (producteurs d'un regard esthétique sur le littoral) ou des premiers touristes aristocrates consommateurs de ces modes naissantes ${ }^{1}$. En privilégiant une interprétation diffusionniste de la «balnéarisation », vue comme un phénomène exogène s'imposant des centres urbains vers la périphérie côtière, on a souvent délaissé l'étude du rôle des populations locales dans la transformation des usages de la plage. Une telle prise en compte des spécificités locales contribuerait pourtant à expliquer les raisons d'un développement différencié du tourisme sur le littoral. Pourquoi certains territoires épousent-ils une vocation balnéaire plutôt qu'une autre ? Pourquoi certaines stations adoptent-elles un modèle de développement touristique plutôt qu'un autre ? Plusieurs travaux récents ${ }^{2} s^{\prime}$ intéressent au rôle des sociétés locales dans la promotion du (ou l'opposition au) développement balnéaire face à d'autres usages du littoral (pêche, agriculture, commerce, conservation, etc.). Selon une approche similaire, cet article aborde le développement touristique d'une destination balnéaire du Nordeste brésilien, Praia da Pipa, en considérant à la fois les trajectoires sociales des catégories dominantes citadines à l'initiative de nouvelles pratiques balnéaires, mais aussi les trajectoires des populations locales (et en particulier leurs élites) ayant pris part à ces nouvelles activités. Quelle relation peut-on établir entre l'émergence d'une économie touristique et les modes de production prévalant avant son développement ? Assistet-on à une juxtaposition d'activités ou à une substitution? Quelles complémentarités ou quels antagonismes peut-on observer dans la transition entre une économie primaire et une économie de services ? L'analyse sociologique proposée ici invite à considérer comment les positions dans leurs espaces sociaux respectifs des différents producteurs du tourisme (notables locaux, propriétaires terriens, élus politiques, agents immobiliers et hôteliers, etc.) ont pu donner lieu à des convergences d'intérêts et à des prises de position favorables au développement balnéaire.
$\mathrm{Au}$ lieu de considérer la plage comme un isolat géographique et un espace à part, cet article envisage l'histoire de la plage dans sa continuité avec d'autres espaces et d'autres usages de l'espace ; en cherchant à comprendre en quoi les transformations à l'œuvre dans l'arrière-pays agraire (les plantations sucrières) et parmi la jeune bourgeoisie urbaine ainsi que les paysans-pêcheurs du littoral ont pu déterminer le développement balnéaire. Dans le cas du Nordeste, le type de relations économiques et sociales entre villages côtiers et fiefs agricoles peut expliquer en partie les trajectoires de développement des territoires balnéaires. Alors que les régions littorales sous l'emprise des élites agraires paraissent plus propices à l'émergence d'un tourisme d'enclaves (type resort, hôtels all-inclusive), du fait notamment de l'adéquation foncière et de l'articulation étroite avec les centres de décision politiques, dans les terres « sans maître » où les populations de paysans-pêcheurs ont conservé une certaine autonomie et un contrôle coutumier sur le système foncier, on observe plus facilement l'apparition d'un tourisme de petites structures intégrées aux villages (la fragmentation
1. Outre l'ouvrage de référence d'Alain Corbin (Le Territoire du vide. L'Occident et le désir du rivage, 1750-1840, Paris, Flammarion, 1990), on peut citer les travaux de Marc Boyer (L'Invention du tourisme, Paris, Gallimard, 1996) et de Jean-Didier Urbain (Sur la plage.
Mœurs et coutumes balnéaires (XIXe XXe siècles), Paris, Payot \& Rivages, 1995), qui ont pour point commun de privilégier une approche littéraire et historique du tourisme balnéaire, centrée sur l'espace des élites urbaines productrices de nouvelles représentations de la plage (lettrés, artistes, villégiateurs, médecins).

2. Johan Vincent, L'Intrusion balnéaire. Les populations littorales bretonnes et vendéennes face au tourisme (1800-1945), Rennes, PUR, 2008 ; Arnaud Sébileau,
"La balnéarisation sous conditions locales. Les concurrences à propos de "l'identité communale" à Saint-Brévin en Pays de Loire ", Mondes du tourisme, 12, 2016, disponible sur : https://tourisme.revues. org/1350\#quotation. 
foncière et l'indétermination des droits de propriété obligeant les entrepreneurs touristiques à nicher leurs établissements dans de petites parcelles ou à négocier leur installation avec une variété d'acteurs locaux). Cette approche théorique des " configurations littorales » empêche par conséquent de prétendre tirer des conclusions générales sur des modèles de développement touristique sans passer par l'étude, d'une part, des trajectoires sociales des agents et de leurs capacités objectives et différenciées à tirer parti du tourisme, et, d'autre part, des « situations ${ }^{3}$ » socioterritoriales donnant à chaque localité sa singularité. Le tourisme ne peut avoir des «impacts » identiques partout, précisément parce que sa définition dépend des producteurs impliqués et parce qu'il s'implante sur des territoires très inégaux.

Raisonner à partir du cas de Praia da Pipa s'avère utile pour réfléchir aux conditions sociales d'émergence du tourisme balnéaire dans le Nordeste brésilien, identifier ses filiations et ses ruptures avec d'autres secteurs de production (plantations sucrières, petite agriculture, pêche, etc.), et le situer par rapport aux grandes transformations sociales du pays au $\mathrm{XX}^{\mathrm{e}}$ siècle. Ancien village de paysanspêcheurs et petit port de commerce situé à proximité d'une zone de plantations sucrières, Pipa est aujourd'hui une station balnéaire cosmopolite et internationale, composée en majorité de petits établissements hôteliers (pour la plupart inférieurs à 50 lits) et d'une grande diversité d'activités (surf, kitesurf, buggy, concerts, festivals, etc.), de visiteurs (régionaux, nationaux et étrangers) et de résidents (autochtones, nouveaux arrivants, résidents secondaires, etc.). Avec près de 6000 habitants permanents et presque autant de lits hôteliers, elle est actuellement la deuxième destination touristique de l'État du Rio Grande do Norte (RN), après Natal, sa capitale [voir illustration 1, p. 46]. Elle fait partie d'une commune (Tibau do Sul) en forte croissance, passée de 3900 à 13300 habitants entre 1970 et 2015.

L'enquête ${ }^{4}$ repose sur une série d'entretiens avec différents acteurs pionniers de la station touristique (anciens villégiateurs, premiers « surfeurs » et hôteliers, élus politiques), complétée par diverses sources documentaires et historiques (registres fonciers et électoraux, ouvrages d'histoire locale, statistiques municipales, etc.). L'étude des trajectoires collectives des principaux groupes en présence (autochtones, néo-résidents, aristocratie rurale) permet de reconstituer l'histoire sociale de la station depuis les premières villégiatures au début du XXe siècle jusqu'à l'époque actuelle en passant par la découverte des spots de surf dans les années 1970, qui marque véritablement les débuts du tourisme dans la région. Elle permet également de comprendre les modalités de « contact» entre ces groupes et les logiques sociales présidant à leur intégration respective sur le marché touristique. Mais avant de s'intéresser à la sociologie des différentes élites en concurrence à Pipa, les deux sections à suivre visent à définir les espaces de la plage et de la plantation, les contextualiser dans l'histoire du Nordeste et comprendre leur articulation socio-territoriale réciproque.

\section{La plage et la plantation}

Le littoral du Nordeste est un espace vaste et complexe, fragmenté dans la longueur entre plateaux sablonneux, plaines dunaires, vallées fluviales (aussi appelées «vallées du sucre ${ }^{5}$ ) et métropoles côtières, et dans sa profondeur entre forêt atlantique, Agreste et Sertão [voir illustration 2, p. 50]. Ces enchevêtrements climatiques et topographiques expliquent en partie la complexité des situations humaines. Du sud de la Bahia jusqu'au Rio Grande do Norte, la Zona da Mata (Zone de la forêt) offre les conditions favorables aux cultures à forte valeur économique destinées aux marchés internationaux (café, cacao, tabac) : à la fois suffisamment proches de l'océan pour profiter des douceurs du climat atlantique et suffisamment éloignées pour être protégées des embruns et bénéficier des sols profonds de l'arrière-pays ${ }^{6}$. À la différence du Littoral Nord, dominé par des champs de dunes qui prolongent les steppes de l'intérieur semi-aride (le Sertão), le Littoral Sud se caractérise par des plages et des falaises verdoyantes typiques de la Zona da Mata. La plage, dans son acception brésilienne, dépasse la simple grève de sable pour inclure l'ensemble des terres côtières caractérisées par des sols peu profonds, souvent instables (du fait de l'érosion côtière et de la mobilité dunaire) et alcalins. Il faut donc distinguer le littoral des plages (pauvre du point de vue agricole) du littoral des plantations qui s'étend jusqu'à l'Agreste (espace de transition entre la Zona da Mata et le Sertão) et où se concentre l'essentiel de la production agraire.

Alors que les plantations de canne à sucre et de café avaient fait la richesse du Brésil colonial, c'est surtout la plage qui s'impose au $\mathrm{XX}^{\mathrm{e}}$ siècle comme espace de développement économique et de distinction sociale. Longtemps considérées comme l'épicentre de la vie culturelle et politique du Nordeste brésilien, les plantations sucrières connaissent un déclin sans précédent à partir de la fin du XIX ${ }^{\mathrm{e}}$ siècle sous l'effet de l'abolition de l'esclavage (1888), de la concurrence internationale et des politiques de " modernisation ${ }^{7}$ " du secteur agricole. La disparition des engenhos - unités de production traditionnelles regroupant la maison de maître (casa grande), les baraquements de travailleurs (senzala) et les cannerais - au profit de grandes raffineries industrielles ${ }^{8}$ (usinas) entraîne un bouleversement radical des rapports sociaux dans la région : le chômage et l'exil de milliers de travailleurs ruraux en direction des villes ${ }^{9}$, les conflits juridiques entre patrons et paysans ${ }^{10}$, les mobilisations syndicales et leur répression (notamment pendant la dictature militaire, de 1964
3. Tristan Loloum, "La situation touristique. Reconfigurations sociales dans une station balnéaire brésilienne ", Tsantsa. Revue de la société suisse d'ethnologie, 20, 2015, p. 163-167.

4. L'enquête est issue d'une thèse de doctorat, voir Tristan Loloum, « Derrière la plage, les plantations. Ethnographie d'une "situation touristique" dans le Nordeste brésilien : le cas de Tibau do Sul, RN", thèse de doctorat en anthropologie sociale et ethnologie, études du tourisme, Paris/ Lausanne, EHESS/Université de Lausanne, juin 2015.

5. Manuel Correia de Andrade, Os Rios do açúcar do Nordeste Oriental, Recife, Instituto Joaquim Nabuco de Pesquisas Sociais, 1957.
6. Manuel Correia de Andrade, A Terra e o Homem no Nordeste, São Paulo, Braziliense, 1963.

7. Peter L. Eisenberg, The Sugar Industry in Pernambuco, 1840-1910. Modernization without Change, Berkeley, University of California Press, 1974.

8. José Sérgio Leite Lopes, 0 vapor do diabo: o trabalho dos operários do acúcar,
Rio de Janeiro, Paz e Terra, 1976.

9. Lygia Sigaud, "Des plantations aux villes. Les ambiguités d'un choix ", Études rurales, 131-132, 1993, p. 19-38.

10. Lygia Sigaud, "Les paysans et le droit : le mode juridique de règlement des conflits ", Social Science Information, 38(1), 1999, p. 113-147. 
à 1985), la perte de prestige et la reconversion des élites agraires ${ }^{11}$, etc. Dans le même temps, la plage, longtemps éclipsée dans l'imaginaire national car considérée depuis la colonisation comme un environnement hostile, sujet aux invasions extérieures, aux intempéries et habité par des populations marginales (pêcheurs, paysans pauvres, indigènes), devient le lieu d'invention de nouvelles pratiques.

Comme en Europe, le « désir de rivage » est d'abord associé aux vertus curatives de l'océan. Les liens établis entre élites brésiliennes et européennes facilitent l'adoption de nouveaux usages. L'anthropologue Paulo Linhares ${ }^{12}$ attribue la diffusion des pratiques balnéaires au Brésil à l'empereur Dom José II, qui inaugure la mode des bains de mer dès la fin du $\mathrm{XIX}^{\mathrm{e}}$ siècle. Certaines villes du Nordeste comme Fortaleza ${ }^{13}$ deviennent particulièrement prisées pour leur ensoleillement et leur air réputés propices au traitement de la tuberculose. À l'hygiénisme des médecins s'ajoute bientôt le romantisme, propagé parmi les cercles de lettrés à l'instar de José de Alencar qui, dans le roman Iracema (1895), idéalise ses héros dans une végétation dense surplombant la mer, à la façon d'un Chateaubriand brésilien. Le tourisme balnéaire devient ainsi une pratique ludique et contemplative étroitement liée au processus de distinction des élites et à la formation des classes moyennes. Àla différence de certains pays d'Amérique centrale et des Caraibes très tôt intégrés aux circuits touristiques internationaux, le tourisme balnéaire brésilien n'est pas qu'une invention exogène, conçue par et pour des étrangers (Nord-Américains ou Européens), mais bien un phénomène national, marqué par des formes particulières de spatialités, de temporalités et de sociabilités ${ }^{14}$.

\section{Configurations littorales}

La complexité territoriale et historique remet en cause le tropisme d'un littoral conçu comme espace à part, homogène et habité par des «populations maritimes »- ou des «peuples de la mer »-dont on serait tenté de naturaliser la singularité. La question du particularisme des populations côtières est ancienne en anthropologie. Elle oppose les approches culturalistes ${ }^{15}$, soulignant la spécificité des modes d'organisation et des systèmes de croyance des sociétés vivant en interaction constante avec la mer, aux fonctionnalistes ${ }^{16}$ qui refusent de voir une spécificité fondamentale des populations maritimes. Ces derniers considèrent en effet qu'il existe une similitude entre les modes traditionnels de production maritimes et paysans dans leurs conditions d'intégration au système capitaliste. L'anthropologue brésilien Antonio Carlos Diegues ${ }^{17}$ distingue quant à lui les « communautés littorales », installées dans d es espaces côtiers relativement protégés et stables du point de vue de l'approvisionnement de ressources (baies, lagunes, estuaires), leur permettant de combiner la pêche avec des activités agricoles; et les «populations maritimes », moins attachées à l'agriculture du fait de la pauvreté du sol et plus souvent obligées de s'avancer au large et de migrer le long du littoral au gré des saisons de pêche. Mais ces distinctions catégorielles ne peuvent occulter le fait qu'en pratique, ces populations ont toujours été confrontées au brassage par le biais des migrations, des mariages, du commerce, etc. L'approche historique permet en ce sens de dépasser les limites de l'approche définitionnelle et fonctionnaliste en soulignant le caractère transitoire et pluriel de ces sociétés, constituées autour de circulations constantes entre les mondes agricoles, maritimes et urbains. Si l'on suit la proposition de Diegues, le cas de Pipa est vraisemblablement à placer du côté des « communautés littorales », dans le sens où l'activité économique du village avant l'avènement du tourisme se partage entre la pêche côtière, la petite agriculture et le commerce maritime. Cette combinaison d'activités, synonyme de liens étroits avec les campagnes de l'intérieur et les centres urbains, renforce l'autonomie de la population locale, sa résilience et son pouvoir de négociation vis-à-vis des élites agraires. Car s'ils ne dépendent pas exclusivement de la mer, les habitants de Pipa ne dépendent pas non plus exclusivement des revenus agricoles.

La nature des rapports entre habitants du littoral et grands propriétaires fonciers de l'arrière-pays semble jouer un rôle déterminant dans le type de développement économique de ces espaces. Les analyses structurales de communautés côtières menées par les anthropologues américains Shepard Forman et Conrad Kottak entre 1960 et 1980 sont particulièrement éclairantes de ce point de vue-là. Bien que décrivant des populations apparemment similaires (deux villages côtiers du Nordeste), ces études témoignent de la grande variabilité des configurations locales en fonction des conditions géographiques et politiques particulières dans lesquelles se trouvent les populations en question. Shepard Forman ${ }^{18}$ analyse une communauté de pêcheurs (Coqueiral) contrôlée de manière autoritaire par des petits chefs locaux [local bigwigs], leaders du syndicat des pêcheurs et planteurs de cocotiers qui monopolisent les échanges commerciaux avec le marché extérieur [voir illustration 3, p. 51]. Au contraire, Conrad Kottak ${ }^{19}$ observe une communauté (Arembepe) dotée d'une forte "idéologie égalitaire » et préservée de la violence politique des élites traditionnelles en raison de son éloignement géographique vis-à-vis des centres de pouvoir . Le désintérêt des élites agraires pour le village d'Arembepe oblige les villageois à organiser la commercialisation du poisson de façon indépendante. À Arembepe, les pêcheurs parviennent à vendre directement leur poisson à la ville, alors qu'à Coqueiral ils doivent passer par des intermédiaires - aux ordres des planteurs - qui ponctionnent l'essentiel des plus-values. Dans les deux cas, la dépendance vis-à-vis des élites
11. Afrânio Garcia Jr., Libres et assujettis. Marché du travail et modes de domination au Nordeste, Paris, Éd. de la MSH, 1989. 12. Paulo Linhares, Cidade de água e sal: por uma antropologia do litoral do Nordeste sem cana e sem açúcar, Fortaleza, Fundação Demócrito Rocha, 1992. 13. Eustógio W. Dantas, Mar a vista: estudo da maritimidade em Fortaleza,
Fortaleza, Edicões UFC, 2011.

14. C'est l'argument du géographe Eustógio W. Dantas dans son livre Maritimidade nos trópicos: por uma geografia do litoral (Fortaleza, Edições UFC, 2009), qui insiste sur le caractère hybride de la " maritimité » brésilienne, constituée à la croisée des modes européennes et des usages traditionnels du littoral brésilien.
15. Aliette Geistdoerfer, Anthropologie maritime : appropriation technique, sociale et symbolique des ressources maritimes, Paris, CNRS, 1989.

16. Raymond Firth, Malay Fishermen. Their Peasant Economy, New York, The Norton Library, 1966.

17. Antônio Carlos Diegues, Maritime Anthropology in Brazil, São Paulo, NUPAUB-USP,
2005, p. 11.

18. Shepard Forman, The Raft Fishermen: Tradition and Change in the Brazilian Peasant Economy, Bloomington, Indiana University Press, 1970.

19. Conrad Phillip Kottak, Assault on Paradise: Social Change in a Brazilian Village, New York, McGraw-Hill, 1992. 


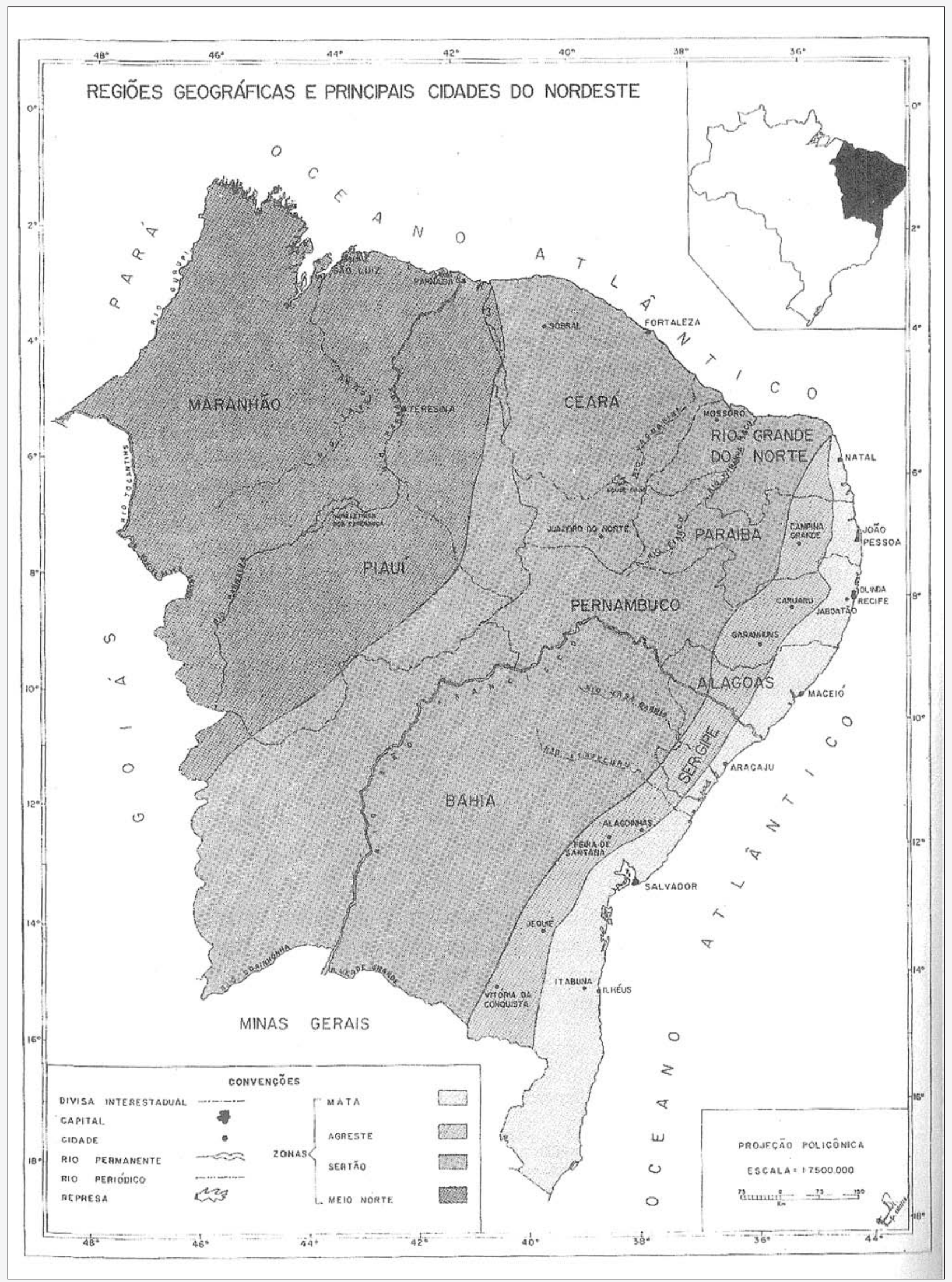

ILLUSTRATION 2. Géographie du Nordeste selon Manuel Correia de Andrade (1963). La zona da mata s'étend du Rio Grande do Norte jusqu'au sud de la Bahia. 


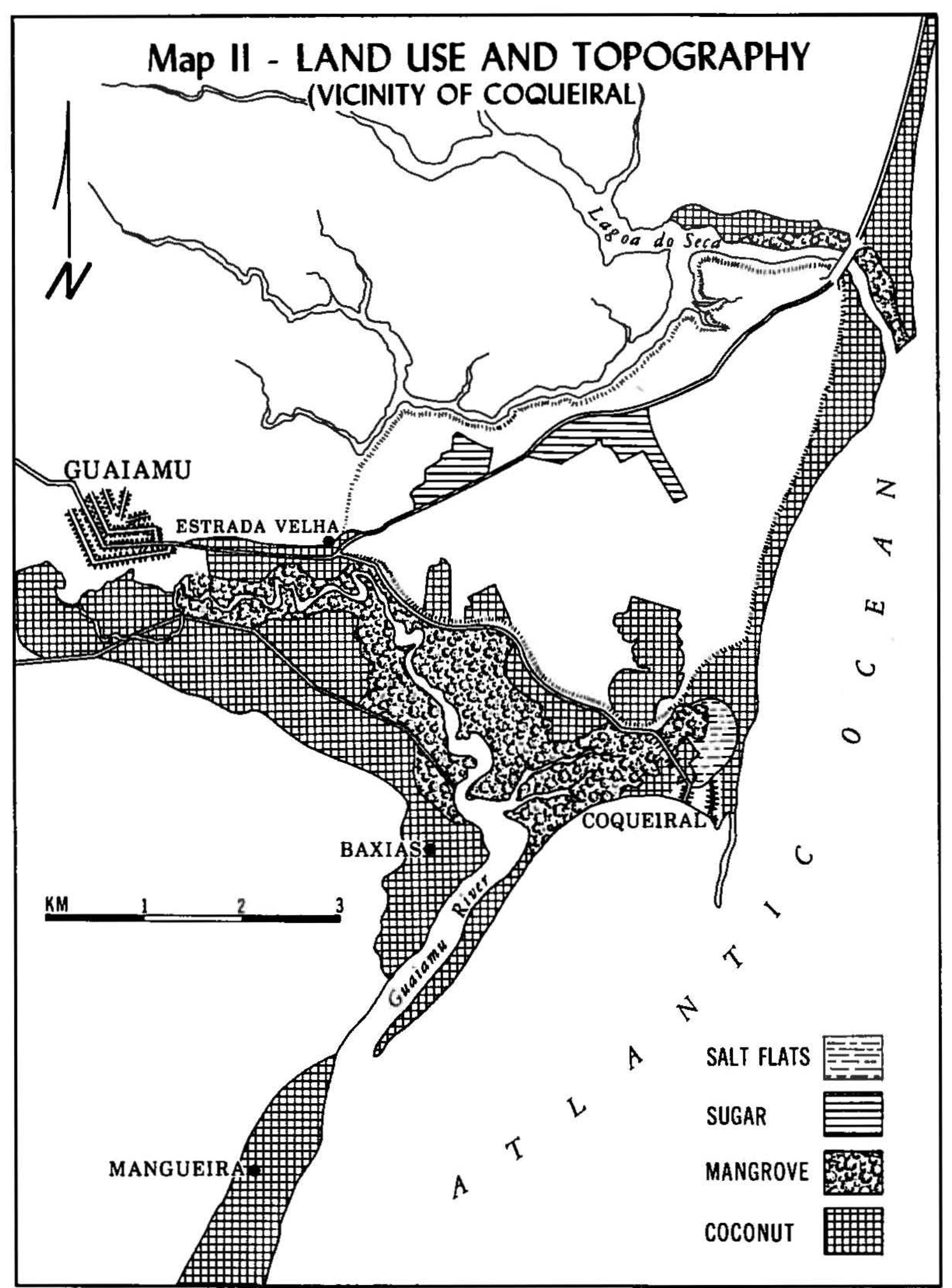

ILLUSTRATION 3. Coqueiral (AL), un village de pêcheurs cerné par les plantations. 


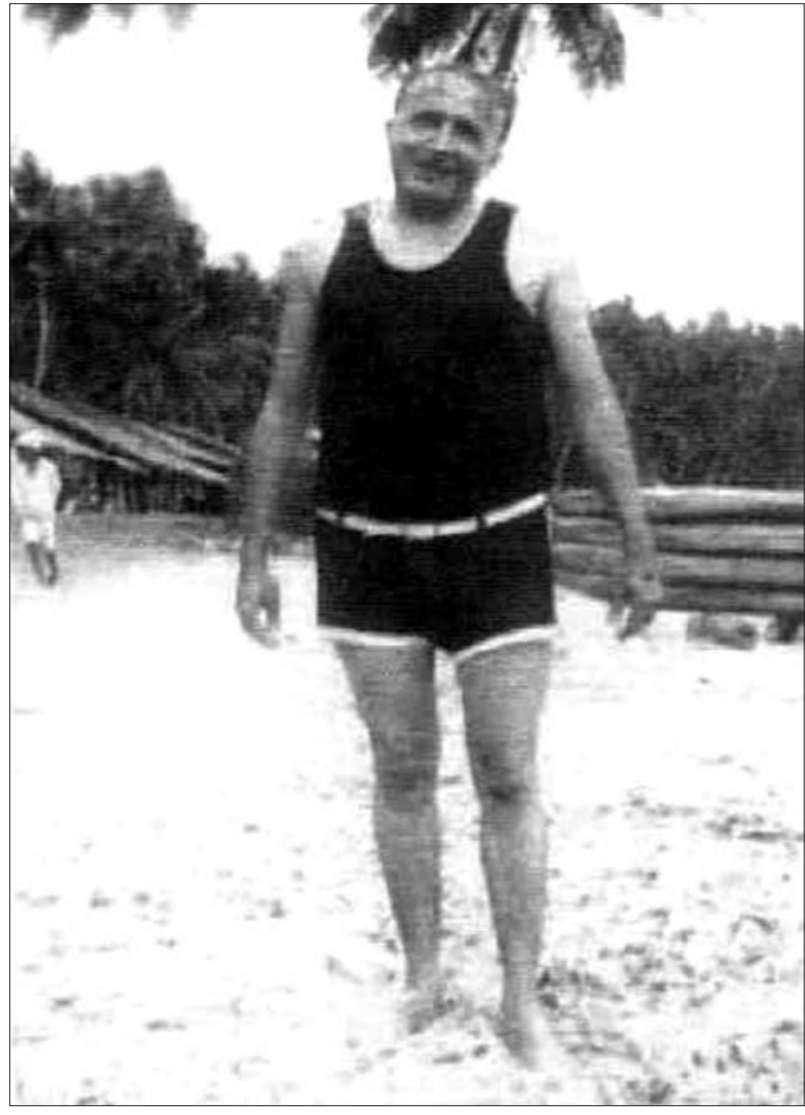

ILLUSTRATION 4. Un maître de plantations à Pipa en 1930.

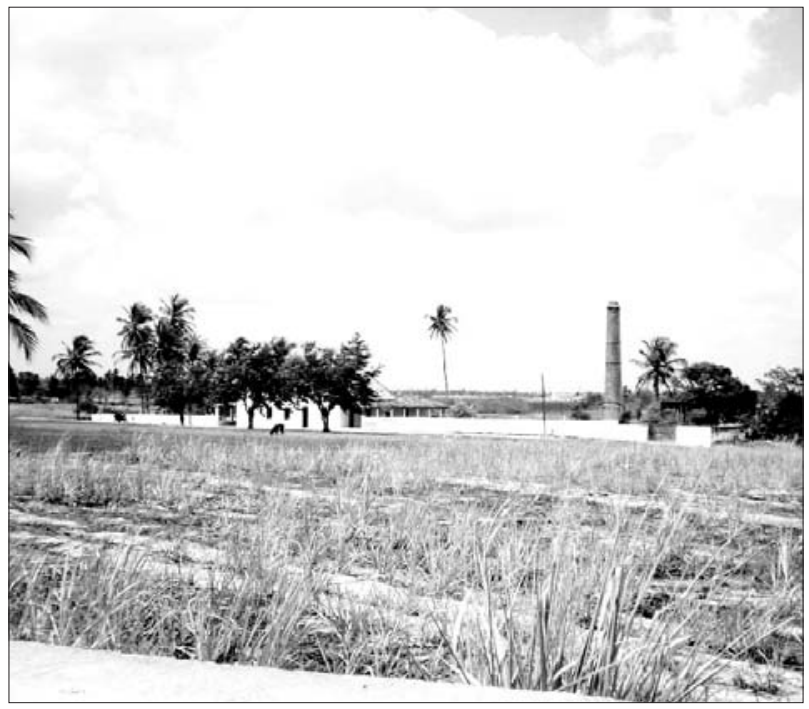

ILLUSTRATION 6. Vestiges de la plantation Benfica, Goianinha, 2014.

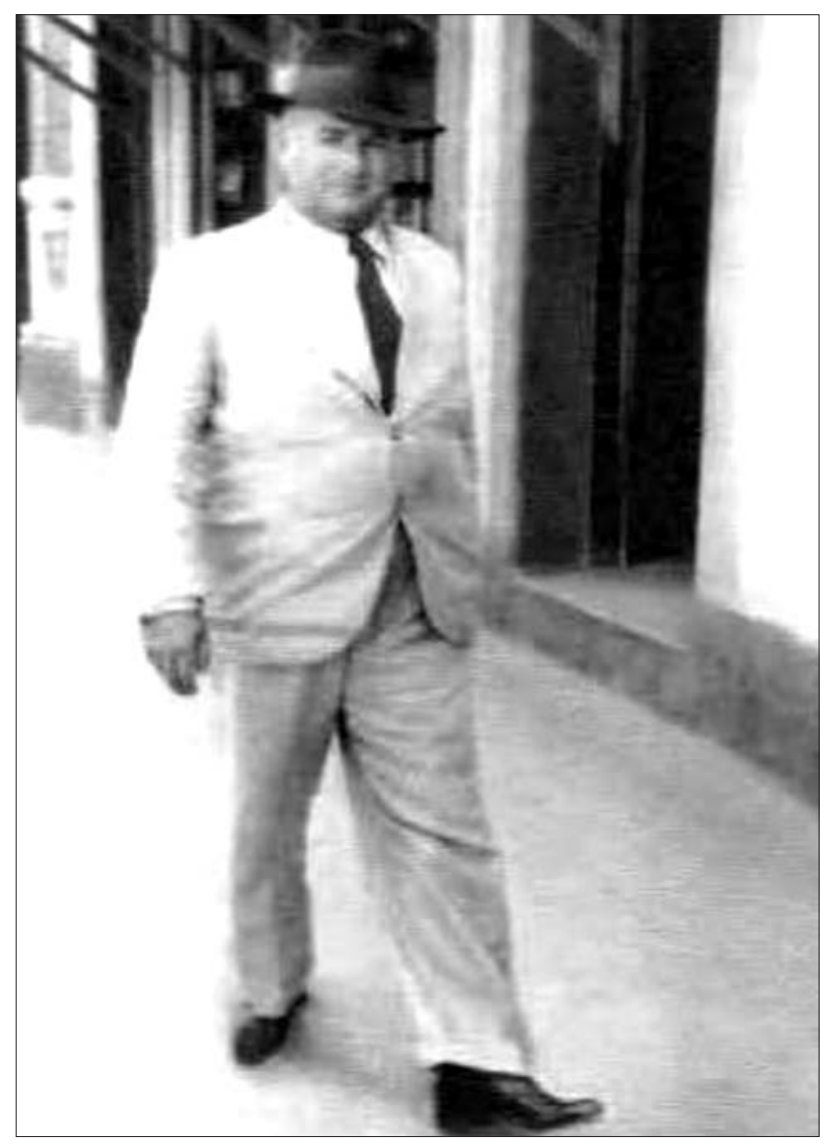

ILLUSTRATION 5. Colonel Odilon Barbalho, initiateur des premières villégiatures de l'élite sucrière de Goianinha en direction de Pipa.

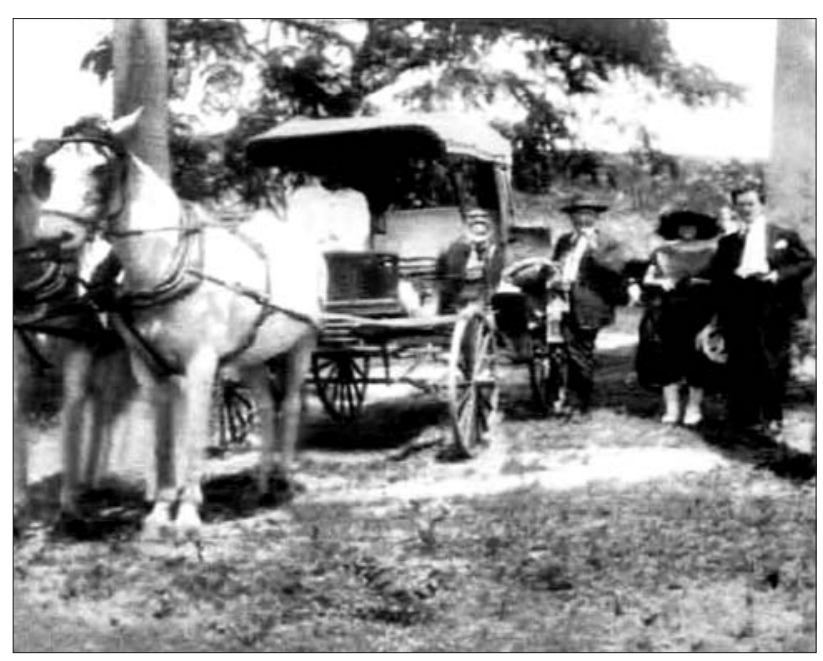

ILLUSTRATION 7. Carrosse de la plantation Ilha Grande, 1930. 


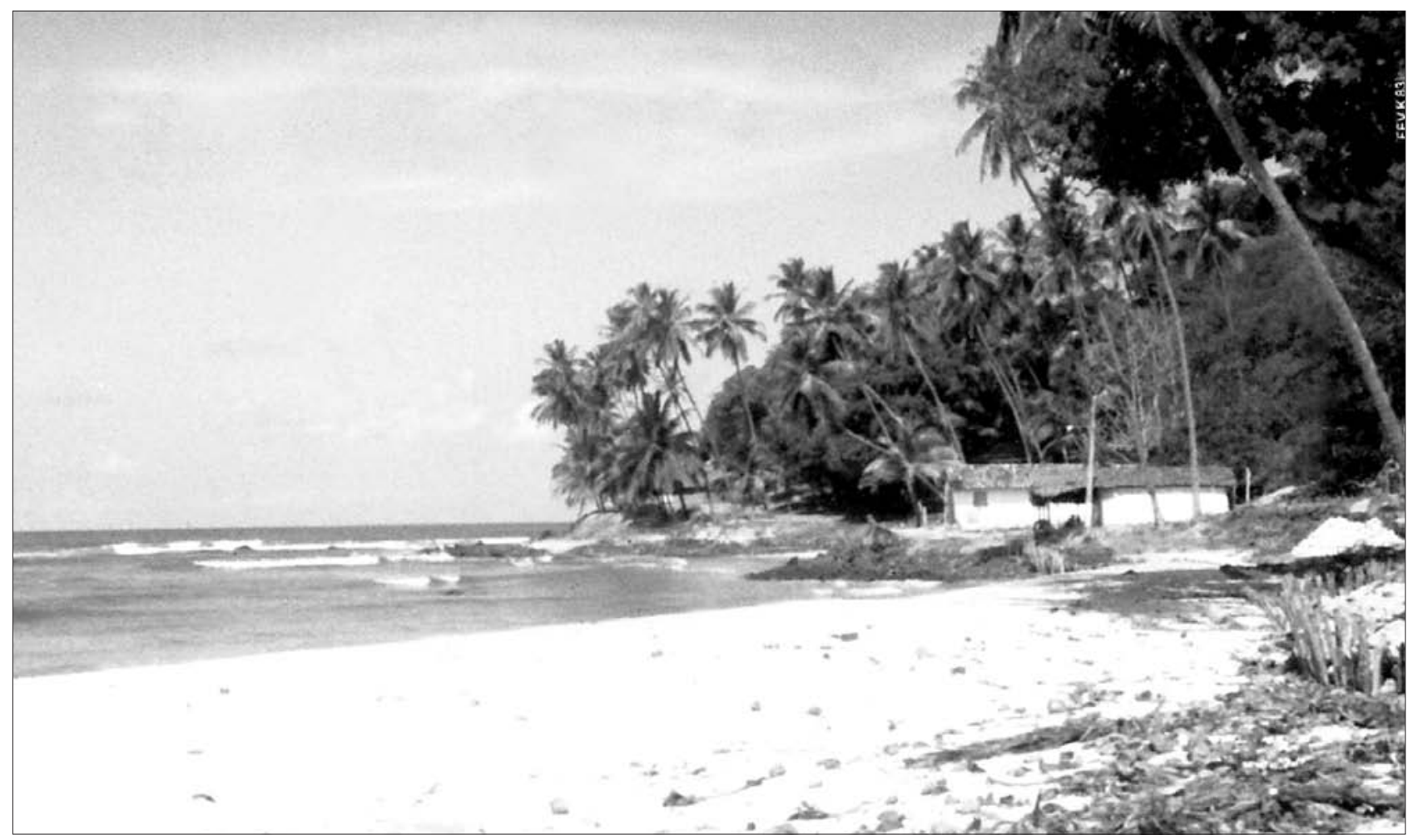

ILLUSTRATION 8. Les maisons de pêcheurs sont peu à peu transformées en villas de vacances, Pipa, 1983.

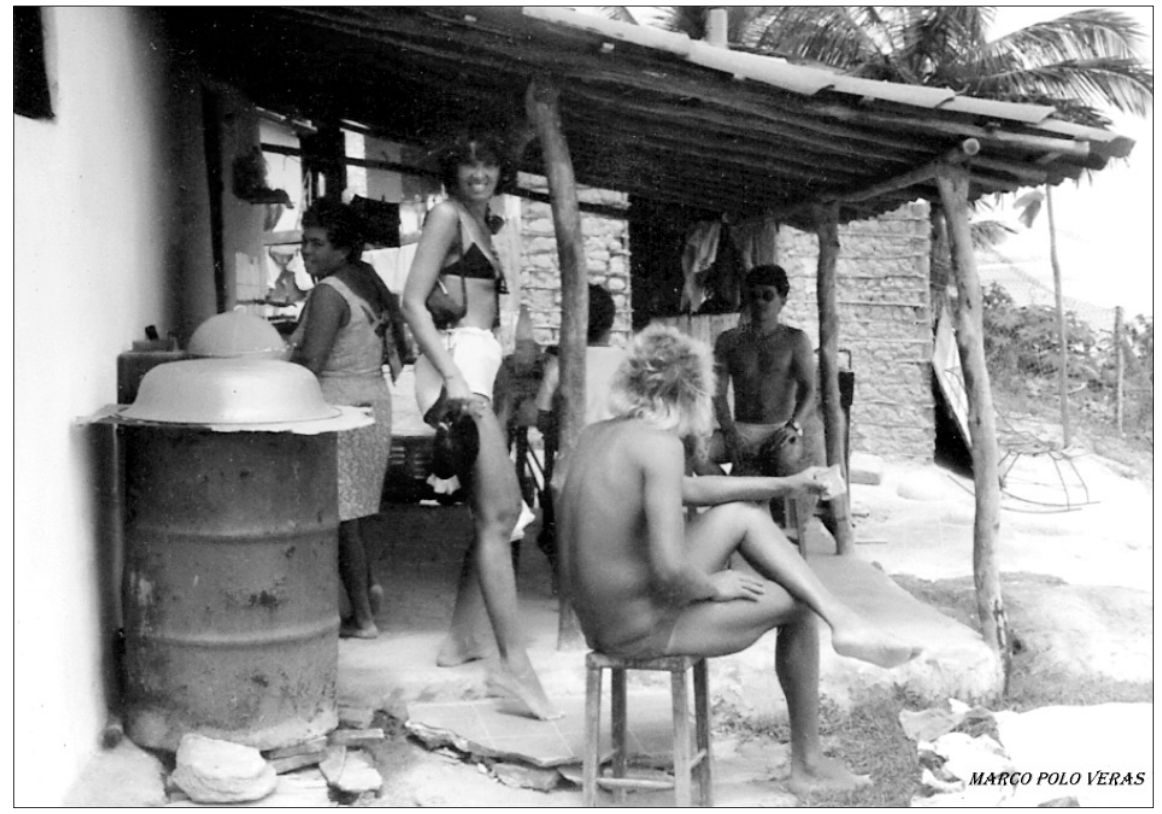

ILLUSTRATION 9. La maison de Dona Eunice, premier restaurant touristique à Pipa, 1987.

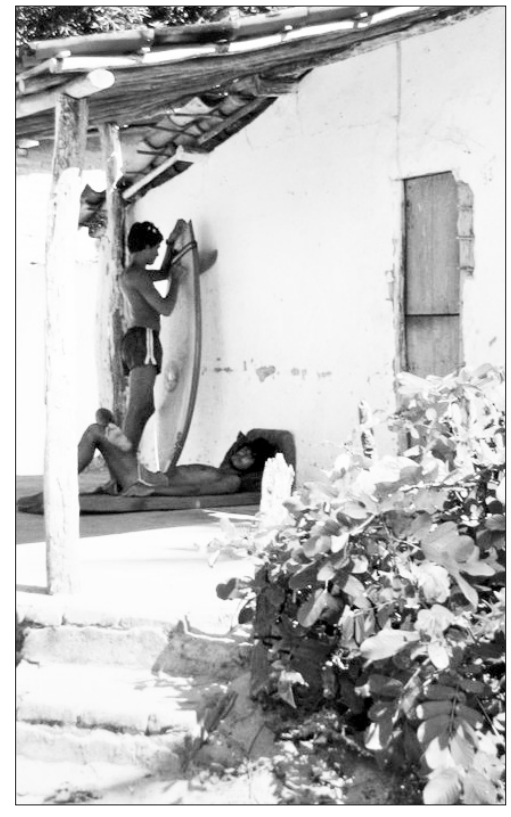

ILLUSTRATION 10. La première génération de surfeurs investit les anciennes maisons de pêcheurs devenues lieux de villégiature des élites sucrières, 1979. 
économiques et politiques locales (grands propriétaires terriens, élus, commerçants) apparaît comme un facteur déterminant dans le comportement des habitants face aux visiteurs extérieurs. Là où les paysans-pêcheurs de Coqueiral semblent avoir intériorisé cette condition d'infériorité et s'adressent aux étrangers comme s'ils s'adressaient à des « seigneurs » (en baissant le regard et par des formules révérencieuses), à l'inverse les habitants d'Arembepe paraissent ne jamais avoir intégré une telle éthique de soumission. Ils cultivent au contraire un esprit irrévérencieux - conforté par une relative indépendance économique - dans lequel vont se reconnaître de nombreux voyageurs hippies des années 1970. Il est à ce titre intéressant de noter qu'Arembepe soit devenu - comme Pipa - une destination touristique prisée de la jeunesse contre-culturelle brésilienne et étrangère, tandis que Coqueiral reste majoritairement dédiée au secteur primaire.

À Pipa, la « bonne distance » vis-à-vis des plantations sucrières confère aux paysans-pêcheurs un statut social singulier, analogue aux « paysanneries marginales " étudiées par Beatriz Heredia $^{20}$ et Afrânio Garcia Jr. ${ }^{21}$. Bien que relégués dans un territoire économiquement pauvre par rapport aux grandes vallées agricoles (sucrières), les paysans-pêcheurs sont en partie préservés de la violence politique et des liens de dépendance personnelle envers les grands propriétaires (fazendeiros) qui n'ont que peu d'intérêt pour cette zone. La possibilité de combiner les revenus de la mer aux revenus de la terre, aussi maigres soient-ils, et de pouvoir commercer avec les métropoles, auxquelles ils sont facilement reliés par voie maritime, constitue également un facteur d'autonomie. Pour Éric Wolf, anthropologue marxiste spécialiste des paysanneries dans le monde, cette association de précarité et d'autonomie des paysans marginaux est une caractéristique essentielle des classes rurales subversives, c'est-à-dire enclines aux soulèvements politiques. C'est ce statut particulier de paysans marginaux (ni vraiment soumis, ni véritablement libérés) qui serait à l'origine de ce que Wolf appelle les « guerres paysannes ${ }^{22} »$. Transposée au tourisme, la situation d'entre-deux des communautés côtières a pu s'avérer propice pour attirer des entrepreneurs « aventuriers » en quête d'espaces préservés, non seulement sur le plan environnemental, mais aussi sur le plan humain, c'est-à-dire des communautés préservées des structures sociales, mentales et politiques héritées des plantations coloniales.

À Pipa, du fait de cette configuration socio-territoriale particulière, le tourisme ne peut déboucher sur une simple reproduction sociale ou sur une extension spatiale de la domination des seigneurs de plantations comme dans d'autres centralités balnéaires constituées sous l'emprise foncière des oligarchies rurales, comme au sud-ouest de l'île Maurice ${ }^{23}$ où le tourisme international est accaparé par l'oligarchie sucrière francomauricienne ; ou encore à Sibaúma ${ }^{24}$, village de descendants d'esclaves situé à quelques kilomètres de Pipa au cœur d'un ancien grand domaine sucrier, où le tourisme apparaît davantage comme « une nouvelle forme de sucre $»^{25}$ tant les inégalités restent profondément marquées. On peut ainsi réitérer l'idée selon laquelle les espaces sous l'influence directe des grands propriétaires ruraux seraient plus propices au développement d'un tourisme composé de grandes enclaves immobilières et hôtelières, à l'image des Integrated Resorts Scheme (IRS) de l'île Maurice, « qui favorisent les grandes propriétés sucrières car cellesci détiennent un capital foncier important, notamment des terres proches du littoral ${ }^{26}$ ». À l'inverse, les petites communautés littorales comme Praia da Pipa, situées à « bonne distance » des grands domaines agricoles, dans des zones moins fertiles, moins accessibles et aussi plus fragmentées sur le plan foncier, voient plus facilement se développer un tourisme de petites structures commerciales avec une forte composante endogène.

\section{Les maitres de plantations à la plage}

Si dans les grandes métropoles brésiliennes la villégiature balnéaire se développe dès le XIX ${ }^{\mathrm{e}}$ siècle $^{27}$, elle ne se généralise dans les campagnes $\mathrm{du}$ Nordeste qu'à partir du début $\mathrm{du}$ $\mathrm{XX}^{\mathrm{e}}$ siècle par le biais de l'aristocratie sucrière qui incarne alors l'élite culturelle de la région [voir illustration 4 , p. 52]. Plusieurs œuvres littéraires témoignent de la fascination nouvelle des lettrés pour le littoral, à l'image de L'Apprenti touriste (1928) inspiré de la visite de l'écrivain moderniste Mario de Andrade à la plantation Bom Jardim, près de Goianinha, ou encore les Lettres de la plage (1967) de l'ethnologue-juriste natif de Tibau do Sul Hélio Galvão.

Ces premières villégiatures ont surtout été documentées par des descendants de cette aristocratie sucrière, à l'image d'Eulalia Barros ${ }^{28}$ ou d'Ormuz Barbalho Simonetti ${ }^{29}$. Cadre de banque à la retraite originaire de Goianinha, ce dernier vit désormais en permanence à Pipa après l'avoir longtemps fréquentée en tant que vacancier, et ce dès son plus jeune âge. Membre de l'une des prestigieuses familles de Goianinha, il a hérité d'une villa située sur la plage centrale de Pipa, aujourd'hui cernée de petits commerces et de baraques. Conteur nostalgique de cet " âge d'or » de la villégiature (avant l'émergence d'un tourisme de masse), il affirme que son grand-père - le « colonel $^{30}$ » Odilon Barbalho, propriétaire de la plantation Benfica située aux abords de Goianinha [voir illustrations 5 et 6, p. 52] - aurait été le premier à s'aventurer à Pipa avec sa famille pour y passer la saison estivale entre janvier et mars $1926^{31} »$.
20. Beatriz Maria Alázia de Heredia, A morada da vida: trabalho familiar de pequenos produtores do Nordeste do Brasil, Rio de Janeiro, Paz e Terra, 1979.

21. Afrânio Garcia Jr., Terra de trabalho: trabalho familiar de pequenos produtores, Rio de Janeiro, Paz e Terra, 1983.

22. Éric Wolf, Les Guerres paysannes du vingtième siècle, Paris, François Maspero, 1974.
23. Emmanuel Grégoire, "Développe- son, A New Kind of Sugar Tourism in the ment touristique et reproduction sociale à l'île Maurice ", Civilisations, 57, 2008, p. 91-106.

24. Tristan Loloum et Cyro Lins, "Land and power: an ethnography of Maroon heritage policies in the Brazilian Northeast", International Journal of Heritage Studies, 18(5), 2012, p. 495-512.

25. Ben R. Finney et Karen Ann Wat-
Pacific, Santa Cruz, Center for South Pacific Studies, 1977.

26. E. Grégoire, art. cit, p. 101.

27. Haroldo Leitão Camargo, Uma préhistória do turismo no Brasil: recreaçaões aristocráticas e lazeres burgueses (18081850), São Paulo, Aleph, 2007.

28. Eulalia Barros, Verdes Campos, Verdes Vales, Natal, auteur, 2004
29. Ormuz Barbalho Simonetti, A Praia de Pipa do tempo dos meus avós, Natal, Nave da Palavra, 2012.

30. Terme utilisé depuis la República Velha (1889-1930) qui fait référence au pouvoir des grands propriétaires ruraux agissant comme dirigeants politiques et chefs de police.

31. 0. Barbalho Simonetti, op. cit. p. 41. 
À l'époque, Pipa n'est encore qu'un village côtier où prédominent la pêche artisanale, la petite agriculture et le commerce maritime. Le récit livré par Ormuz Simonetti de cette première migration estivale laisse deviner la verticalité des rapports entre planteurs et pêcheurs. Le Colonel Odilon mandate un messager pour demander à son " compère ", Antonio Pequeno, l'un des chefs du village, de faire ouvrir un chemin à travers la dense végétation du plateau jusqu'à la route la plus proche afin de permettre le passage des diligences des vacanciers [voir illustration 7, p. 52]. Honorés par la présence d'aussi illustres visiteurs, certains pêcheurs cèdent gracieusement leur maison [voir illustration 8, p. 53] le temps de la belle saison :

\begin{abstract}
"Les maisons occupées par les estivants appartenaient aux pêcheurs qui, la plupart du temps, quittaient leurs foyers pour les louer ou même les prêter à ceux qui arrivaient. Ils allaient vivre temporairement chez des parents. C'était des maisons très pauvres, construites avec du bois local recouvert de torchis [taipa] et surmontées de paille de cocotier ${ }^{32}$. "
\end{abstract}

Il s'instaure entre estivants issus de l'aristocratie sucrière de l'arrièrepays et paysans-pêcheurs du littoral une relation de domination teintée d'amitié et de loyauté. À Pipa, ces rapports se manifestent par des liens de « compérage » entre les seigneurs ruraux et leurs subalternes : en acceptant de devenir parrains (ou marraines) d'enfants autochtones, les nobles estivants deviennent ainsi les compères (ou commères) des pipenses. Rituel religieux «solennisé en public et devant Dieu " ${ }^{33}$ à l'occasion du baptême, le compérage est une parenté symbolique qui institue des liens d'obligations réciproques entre des familles aux positions sociales très inégales. Courantes à l'époque dans les campagnes nordestines ${ }^{34}$, ces formes d'interdépendance morale et hiérarchique se traduisent par une succession de dons et contre-dons toujours inégaux. Les maîtres de plantations font profiter aux pêcheurs et agriculteurs de quelques-uns des privilèges afférant à leur statut (accès aux soins médicaux primaires, démarches administratives, donations à la paroisse locale, etc.) tandis que ces derniers font preuve d'une hospitalité zélée, d'une loyauté politique ou d'une dévotion personnelle indéfectible à l'égard des familles d'estivants. C'est notamment au titre de cette " amitié » que les vacanciers vont progressivement acquérir certaines maisons du bord de mer à des prix défiant toute concurrence. À Pipa, plusieurs personnes racontent que certains terrains auraient même été échangés contre une bicyclette ou un réfrigérateur.

Mais l'emprise de l'élite sucrière sur les populations du littoral doit toutefois être relativisée, notamment au regard des modes de domination alors en vigueur dans le huis clos des plantations, où les seigneurs détiennent un pouvoir de vie et de mort sur leurs ouvriers $^{35}$. L'anthropologue américain Shepard Forman distingue en effet la « dépendance patronale » d'une part, où les assujettis n'ont d'autre choix que de servir un individu en position dominante, et le « clientélisme patronal » d'autre part, où les dominés ont le choix entre plusieurs « bienfaiteurs", et donc une certaine marge de négociation $^{36}$. Tout semble indiquer que Pipa se trouve dans le deuxième cas de figure, car la domination des seigneurs de plantations sur les paysans-pêcheurs s'y exerce de façon partielle, distante et intermittente. Partielle, car les grands propriétaires fonciers du continent ne comprennent pas tout de suite l'intérêt financier de l'acquisition de terres côtières. Les estivants demeurent de fait longtemps concentrés dans un seul et même quartier - le « quartier des estivants »- situé au centre du village, à côté de l'église et en première ligne de côte. Rien ne laisse alors présager $\mathrm{du}$ développement exponentiel du tourisme au-delà des centres urbains. La villégiature reste, jusque dans les années 1970, un phénomène marginal, réservé aux riches familles des environs qui cherchent dans l'activité balnéaire l'occasion de renouveler un entre-soi. Cette domination est aussi distante et intermittente, car la présence des maîtres de plantations sur le littoral se limite essentiellement aux vacances d'été (de décembre à février) et à la Semaine sainte (avril). Le reste de l'année, leur vie tourne autour de la plantation et de la ville. Et surtout, les terres de Pipa, trop pauvres pour susciter l'intérêt de l'État ou des grands propriétaires ruraux, sont un amas de petites parcelles aux contours et au statut juridique incertains, si bien que la zone côtière demeure en dehors de la zone d'influence directe des grandes élites agraires. Cette position marginale des populations de la côte - ni misérables ni opulentes, ni soumises ni dominantes - va trouver un écho à partir des années 1970 chez un certain nombre de jeunes urbains en quête de nouveaux modèles culturels.

\section{Les « surfeurs » comme avant-garde touristique}

Bien que les premières villégiatures à Pipa remontent au début du $\mathrm{XX}^{\mathrm{e}}$ siècle, c'est véritablement dans les années 1970 et 1980 que le tourisme commence à s'y développer, sous l'impulsion de jeunes surfeurs qui vont faire connaître ses spots de surf au monde entier. La découverte de Pipa par les surfeurs est un véritable mythe fondateur et beaucoup d'entrepreneurs touristiques de la station se revendiquent encore de cet héritage, même lorsqu'ils n'ont qu'une relation très anecdotique avec le surf. Ce recours à l'univers du surf comme argument de légitimation évoque les travaux du sociologue Christophe Guibert ${ }^{37}$ sur l'usage opportuniste de la catégorie « surfeur », qui tend à gommer l'origine sociale favorisée des premiers pratiquants tout en leur conférant les profits symboliques d'un style de vie associé à la contre-culture, au voyage, à la nature et à l'ascétisme de la pratique. Le style anticonformiste des surfeurs a souvent été interprété comme une forme non conventionnelle d'expression politique, dans un pays en proie à la répression des mouvements d'oppositions et à la censure des canaux traditionnels de diffusion des idées politiques (livres, places publiques), tant sous l'Estado Novo de Vargas (1937-1945) que sous la dictature militaire (1954-1985).
32. Ibid., p. 42.

33. Jeremy Boissevain, "Patronage in

Sicily", Man, 1(1), 1966, p. 18-33.

34. Ellen F. Woortmann, Herdeiros, paren- tes e compadres, São Paulo, Hucitec/E

DUnB, 1995.

35. A. Garcia Jr., Libres et assujettis..., op. cit.
36. Shepard Forman, The Brazilian Peasantry, New York, Columbia University Press, 1975, p. 69

37. Christophe Guibert, « Le premier âge du surf en France : un sport socialement sélectif ", Science \& Motricité, 61, 2007, p. 89-100, en particulier p. 95. 
Il n'en reste pas moins socialement sélectif et culturellement conforme à des modèles dominants.

Les années 1970 et 1980 sont propices à l'émergence de nouvelles pratiques de loisirs et de nouvelles vocations professionnelles parmi la jeunesse favorisée brésilienne. Le Brésil s'est engagé depuis Vargas dans un long processus d'industrialisation, de centralisation du pouvoir, de réunification territoriale du pays et de refondation de la « conscience nationale » initié dans les années $1930^{38}$. Ces nouvelles dynamiques encouragent les circulations entre les différentes régions et font $\mathrm{du}$ littoral un nouvel enjeu stratégique (pour l'industrie, le transport, l'exploitation du pétrole et la défense du territoire). Le Nordeste, mis en avant dans la littérature régionaliste de la première moitié du $\mathrm{XX}^{\mathrm{e}}$ siècle comme berceau de l'authenticité rurale et de l'identité nationale, attire alors beaucoup de jeunes voyageurs du sud-est urbain et industriel. Par ailleurs, le rapprochement avec les États-Unis pendant la Seconde Guerre mondiale et l'installation de nombreuses bases militaires américaines sur le littoral brésilien sont le signe d'un changement d'influence : les normes européennes cèdent le pas au modèle culturel américain, dont sont issus le surf et le mouvement hippie.

Dans l'État du Rio Grande do Norte, la première pratique du surf remonte aux années 1960 et est attribuée à deux frères originaires de Rio de Janeiro (point de départ du surf au Brésil), Marquinhos et Rosset, fils d'un militaire carioca muté à Natal. Étudiants dans un riche lycée privé, ils transmettent rapidement leur passion à d'autres jeunes issus des milieux aisés de la ville. Fils de militaires, de magistrats, de médecins, de professeurs ou de cadres, les « surfeurs » fréquentent les mêmes lycées privés que les enfants de planteurs venus se former en ville, à l'image de Luruca, réputé pour être l'un des premiers "découvreurs» du surf à Pipa :

\footnotetext{
«Pipa avant, c'était un petit coin perdu au milieu de nulle part, maintenant regarde ce que c'est devenu : une référence nationale, avec un métissage
}

de cultures, du folklore et plein de bons spots de surf. Je suis un surfeur de la première heure, la première fois que je suis venu c'était pour la Semaine sainte de 1978. Je suis vite devenu un habitué, je dormais toujours sous les vérandas des maisons de vacances, jusqu'à ce que je me marie avec une "locale" et que je devienne habitant. Certains surfeurs, des amis à moi, venaient depuis 1975 environ. L'un d'entre eux, Luruca (ou Loremberg de Natal) sortait avec la fille de Cirene Simonetti, qui était d'une famille très importante. II [Loremberg] est venu passer un été avec la fille en question, il a tout de suite vu que c'était un excellent coin pour surfer. C'est à partir de là qu'il a commencé à en parler à ses amis qui l'ont tout de suite rejoint. L'année suivante il y avait déjà des jeunes d'autres États qui affluaient... » [Lucrécio, ancien surfeur, résident de Pipa].

À Pipa, l'intrusion de ces nouveaux acteurs est l'occasion d'une profonde reconfiguration des rapports sociaux. Malgré le fossé social et culturel existant entre jeunes bourgeois des villes et paysans-pêcheurs du littoral, il se tisse entre eux une affinité bien différente de l'amitié très hiérarchique qui liait les familles autochtones aux premiers villégiateurs. Les jeunes surfeurs se formalisent moins que les estivants traditionnels des distinctions de statut et du confort matériel. Alors que ces derniers débarquaient chargés de bagages et de denrées pour tenir durant tout l'été, les surfeurs eux voyagent légers. À la différence des estivants, ils ne viennent pas avec leurs employés domestiques, mais font appel plutôt à des locaux pour préparer leurs repas quotidiens, à l'image de Dona Eunice qui transforme sa maison en restaurant pour surfeurs [voir illustration 9, p. 53]. Ils ne viennent pas non plus en famille pendant les grandes vacances, à l'instar des villégiateurs, mais plutôt entre amis, pendant les longs weekends prolongés qu'offre la vie étudiante. $\mathrm{Au}$ début, ils campent indifféremment sous les auvents des villas inhabitées des estivants, dans les jardins des locaux, voire à même la plage [voir illustration 10, p. 53]. Contrairement aux villégiateurs traditionnels, encore empreints des modes ruraux de sociabilité, les surfeurs ne partagent pas vis-à-vis des locaux le même sentiment de supériorité que l'aristocratie agraire. Alors que les estivants veillent à maintenir une forme de ségrégation entre l'habitat autochtone (concentré au centre du village) et le « quartier des estivants » (s'allongeant en bord de mer), les nouveaux arrivants, loin d'opérer une éviction des couches populaires, vont plutôt chercher à en gouverner la diversité sociale ${ }^{39}$.

Ils portent un regard nouveau sur le style de vie des populations du littoral. La « simplicité » des habitants autochtones n'est pas vue comme une marque d'infériorité, mais plutôt « d'authenticité ». Au-delà du seul panorama côtier, la communauté locale devient elle-même un objet d'attraction [voir illustration 11, p. 59]. C'est en partie cet « art de vivre local» (le jeitinho nativo), anti-modèle de la ville impersonnelle et chaotique, qu'ils sont venus rechercher. Leur intérêt se porte autant sur la plage que sur le centre du village [voir illustration 12, p. 59], où certains commencent à acquérir du foncier. Leur proximité avec les locaux leur permet d'acheter à prix d'ami et laisser leur terrain sous bonne garde en leur absence :

\begin{abstract}
" J'ai tellement aimé l'endroit, la culture locale, que j'ai décidé d'acheter un petit lopin de terre avec l'idée de peut-être m'installer un jour. II faisait environ 1,5 hectare. À l'époque la terre ne valait rien, tu pouvais même échanger un terrain contre une télévision, un frigo. J'habitais à Rio. Un an après je suis revenu avec ma femme, et comme je n'avais pas d'argent à l'époque pour faire une auberge, j'ai ouvert un petit bar avec un ami autochtone [nativo]. On l'a laissé en charge du bar et on est retourné à Rio. C'était déjà lui qui s'occupait de la propriété en mon absence, je le payais pour qu'il surveille mon terrain. Ce n'est que 15 ans après que je suis venu m'installer et qu'on a démarré les bungalows. " [Heitor, originaire de Rio de Janeiro, publicitaire et hôtelier].
\end{abstract}

Dans les années 1980, la dissémination du nom de Pipa à travers le surf attire de nouveaux publics, moins intéressés par le surf que par la beauté des paysages, le cadre de vie et la culture locale.
38. "Au début du XXe, nombre d'intellectuels brésiliens partageaient le sentiment de l'inexistence d'une culture nationale. [...] On assiste ainsi à l'institutionnalisation progressive du souci de former la "conscience nationale", grâce à l'implantation d'un marché éditorial dans le pays et au développement du système d'enseignement qui a permis la diffusion des nouveaux schèmes de pensée liés à la modernisation. Leur apport le plus spécifique fut sans doute la restauration de l'image de soi, substituant au débat obligé depuis le XIXe sur l'infériorité d'un peuple de métis l'image d'une nation jeune et porteuse d'avenir ». Voir Afrânio Garcia Jr., " Les intellectuels et la conscience nationale au Brésil », Actes de la recherche en sciences sociales, 98, 1993, p. 20-33, en particulier p. 20.
39. À la manière des gentrifieurs qui investissent les quartiers populaires des grandes villes occidentales, voir Sylvie Tissot, De bons voisins. Enquête dans un quartier de la bourgeoisie progressiste, Paris, Raisons d'agir, 2011. 
Voyant là l'opportunité de vivre de leur passion, certains songent à s'installer définitivement à Pipa et démarrer une activité touristique (hôtel, restaurant, excursions, etc.). Il s'agit généralement d'anciens surfeurs ou de voyageurs de la première heure revenant sur les itinéraires vacanciers de leur jeunesse, désormais en couple ou en famille et avec les moyens de leurs ambitions. Issus d'un milieu aisé, le plus souvent diplômés du supérieur, ils disposent non seulement d'un capital financier, obtenu au cours de métiers antérieurs ou auprès de leur famille, mais aussi d'une sensibilité culturelle particulière - le style rustique et décalé des jeunes bien élevés faisant mine de ne pas l'être - nourrie par un environnement social favorisé et l'imprégnation des modes contreculturelles. Le tourisme et l'hôtellerie apparaissent ainsi comme une façon de valoriser économiquement ce style de vie " alternatif » en accomplissant le rêve d'une sédentarisation à la plage. On retrouve ici l'hypothèse de reconversion sociale de l'avant-garde bourgeoise formulée par Bertrand Réau au sujet des inventeurs du Club Med et du tourisme d'aventure. En retraçant la genèse sociale de ces nouvelles formules de vacances, Réau montre que leur succès repose essentiellement sur l'homologie structurale entre les avantgardes bourgeoises investissant leurs goûts pionniers dans l'offre de loisirs et les attentes des touristes en quête de pratiques distinctives : « La création de nouvelles positions sur les marchés des loisirs, des vacances et du tourisme semble être une "spécialité" des avantgardes bourgeoises et/ou aristocrates qui commercialisent avec succès leur mode de $v^{4} e^{40}$. »

\section{Les reconversions des descendants de planteurs}

Une autre hypothèse avancée par Bertrand Réau est que le tourisme est l'occasion pour certaines catégories bourgeoises et/ou aristocrates de s'inventer de nouvelles voies d'autopromotion sociale lorsque les débouchés ordinaires commencent à faire défaut : "En "avance" sur les goûts de leur classe sociale, l'investissement de leur "sensibilité culturelle" dans l'espace des loisirs et du tourisme apparaît comme un moyen de se reclasser $^{41}$ ». Dans le Nordeste, c'est particulièrement le cas de certains descendants de l'aristocratie sucrière qui se retrouvent confrontés à la crise des engenhos et à la profonde restructuration du secteur agricole. Pour les enfants de planteurs, les métiers de cadre, d'ingénieur et les fonctions politiques régionales ou nationales (après un passage obligé par l'université) sont les voies de reconversion les plus courantes de reclassement ${ }^{42}$. Mais le tourisme est aussi l'occasion de réaffecter le capital familial : capital foncier émanant des propriétés de vacances ou rurales, capital culturel acquis à l'université et au cours des voyages, capital social, enfin, issu de la longue fréquentation des populations et des institutions locales. Parmi les entrepreneurs touristiques rencontrés à Pipa, le grand nombre d'hôteliers formés en agronomie semble confirmer l'hypothèse d'une complémentarité entre les carrières agricoles et touristiques parmi l'élite régionale.

L'itinéraire de Geovani, propriétaire de l'un des principaux hôtels de Pipa, est à ce titre emblématique des trajectoires de conversion de l'élite locale. Né en 1960, initié au travail de la canne par son père (fournisseur de la raffinerie voisine), découvrant le tourisme dans sa famille qui avait coutume de passer ses vacances sur les plages de la région, il est marié à une notaire de la ville voisine qui est, elle aussi, issue d'une famille de l'élite sucrière locale. Dans les années 1980, il profite des subventions publiques offertes par le programme Pro-Alcool pour acquérir à bon prix des terres agricoles proches de la côte, autrefois impropres aux cultures intensives. Programme agroindustriel mis en œuvre à partir de 1975 pour répondre aux chocs pétroliers par la production d'éthanol, le Pro-Alcool permet aux propriétaires d'assumer les surcoûts liés à la fertilisation chimique des sols. Il participe ainsi à l'extension spatiale des cultures de canne à sucre, notamment en direction des terres moins fertiles de la côte :

"Quand j'ai terminé la faculté, à 22 ans, je travaillais déjà des terres depuis 18 ans. [Des terres de votre famille?] Non. II y a une terre que mon père m'a donnée, le reste j'ai acheté. J'avais besoin d'étendre ma canne à sucre, acheter plus de terre, et par hasard il y avait ces terrains à vendre, là où j'ai la fazenda. 236 hectares. Quand j'ai acheté, un des propriétaires m'a dit "regarde, si tu veux le terrain donne sur la plage". Je connaissais déjà l'endroit donc que je lui ai demandé de me montrer. J'ai commencé à travailler la partie agricole derrière, en plantant de la canne. Puis quand je me suis marié, en 1985, j'ai commencé à voyager avec mon épouse. On a connu Porto Seguro, qui était le début du tourisme au Brésil, puis llheus... C'était les débuts de "la plage" au Brésil. Donc je me suis dit "Pipa, c'est un lieu qui va marcher". En 1989 ou 1988, il y a eu la première vraie auberge à Pipa, Pousada da Gaivota, et ensuite le Village Natureza. En 1989 j'avais déjà l'intention de m'installer, mais je n'ai pas pu à ce moment-là. Pipa a continué de se développer et j'ai finalement ouvert l'hôtel en 1994. II y avait un gros besoin de lits à l'époque. En quelque sorte, je faisais partie de la deuxième vague de pionniers. " [Geovani, hôtelier à Pipa, fils de planteur].

Rogério Bivar Simonetti est un autre exemple de réinvestissement des ressources traditionnelles de l'élite agraire dans le tourisme. Propriétaire d'un hôtel de bord de mer depuis 1992, il a été l'un des premiers hôteliers à s'installer dans la région, en même temps que quelques surfeurs et expatriés. Âgé d'une cinquantaine d'années, il est un cousin d'Ormuz Simonetti, arrière-petit-fils d'un maître de plantations « colonel » et estivant de la première heure à Pipa. Bien que sa famille soit originaire de Goianinha, il est né à Recife après que son père ait épousé une femme de "bonne famille » dans le Pernambuco. Comme Geovani, il est formé en agronomie et a gardé de son héritage familial un profond attachement à la terre, qu'il manifeste à travers un goût prononcé pour les chevaux et les vaquejadas, sorte de corrida brésilienne pratiquée à cheval et consistant à capturer une vache en la tirant par la queue. Face à l'impossibilité de combiner ce sport - qui a lieu les week-ends - avec son activité hôtelière, il se reporte sur les randonnées équestres, qu'il vend sous forme d'excursions aux clients de son hôtel. Dans les années 1990, il aménage un ranch à la périphérie 
de Pipa dans une propriété achetée à un parent. En 2000, alors qu'il possède plus de 25 chevaux, il décide de se lancer dans l'élevage, plus par passion que par nécessité, précise-t-il. Il transforme peu à peu son ranch en haras spécialisé dans le Mangalarga Marchador, une race de trotteurs typiquement brésilienne et particulièrement adaptée à la randonnée. Depuis, Rogério partage son temps entre les concours équestres, les randonnées et son hôtel. Il est aujourd'hui l'un des principaux entrepreneurs de Pipa et un éleveur de chevaux reconnu. $\mathrm{Sa}$ trajectoire indique comment un capital agraire (économique et culturel) peut être reconverti dans le tourisme et les loisirs. Son accès privilégié au marché foncier local lui a permis d'acquérir non seulement un terrain en bord de mer, espace traditionnellement investi par les estivants depuis le début du siècle, mais aussi un ranch de plusieurs dizaines d'hectares à quelques kilomètres de Pipa, dans une zone de latifundia encore occupée par la canne à sucre et l'élevage, et qui constitue aujourd'hui un nouveau front d'expansion urbaine pour la ville touristique. En effet, après un développement dense autour du centre-ville, on commence à voir se développer depuis une dizaine d'années quelques grands lotissements " ex-urbains » à la périphérie de Pipa, profitant des grandes parcelles agricoles d'un seul tenant [voir illustration 13, p. 60], pré carré séculaire de l'aristocratie rurale reclassé en zone à bâtir. C'est aussi la resignification d'un capital culturel, comme les sports équestres, symboles de l'héritage colonial et du pouvoir des barons ${ }^{43}$. Ou encore les vaquejadas, rite de prestige rappelant le travail de conduite du bétail dans les campagnes nordestines, traditionnellement pratiqué par les éleveurs lettrés ${ }^{44}$ et faisant l'objet dans sa forme contemporaine d'un véritable négoce culturel, avec ses professionnels, ses investisseurs, ses émissions TV et ses entreprises spécialisées ${ }^{45}$.

Les descendants de l'élite agraire restent influents dans la région - notamment du fait de leur accès privilégié aux institutions publiques fédérées et fédérales, parmi lesquelles ils disposent d'importants réseaux (sociaux, familiaux, politiques) - mais leur influence paraît nettement en déclin par comparaison avec l'ascendant moral et politique dont pouvaient jouir leurs aïeuls. Cette déprise est d'abord sociale et territoriale : l'ancienne aristocratie agraire locale se délite sous l'effet des mutations du monde rural et doit se reconstituer ailleurs. Pour les villégiateurs traditionnels, l'arrivée de nouveaux résidents est perçue comme une menace à leur quiétude et à leur monopole sur la plage. C'est du moins ce que laisse entendre cette anecdote au sujet de la première auberge ouverte par une étrangère (Barbara, une voyageuse allemande qui rachète au début des années 1980 le terrain d'un membre de la famille Barbalho endetté) dans le quartier des estivants : " Tout le voisinage s'est mis à me détester, j'étais l'intruse - une femme qui plus est ! Le premier élément étranger à venir troubler leur quiétude... »[Barbara, hôtelière allemande, résidente à Pipa]. Parmi les familles traditionnelles d'estivants, beaucoup finissent par vendre leurs propriétés : au fil des successions, à mesure que la composition sociale de la plage change et que l'immobilier s'enchérit. Le quartier des estivants, où sont concentrées les villas de vacances des familles de planteurs, se transforme progressivement en quartier touristique. Sur la promenade, la multiplication des baraques de plages [voir illustration 14, p. 61], pour la plupart tenues par des entrepreneurs autochtones, marque le début de la fin pour les villégiateurs d'antan :

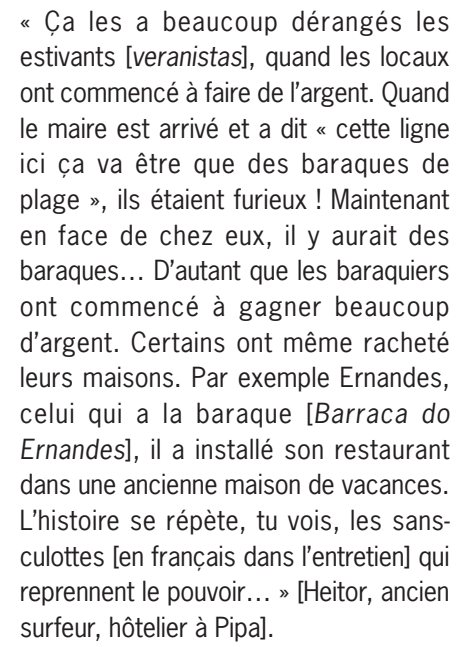

Certains villégiateurs tentent bien de résister à travers d'éphémères mobilisations - que l'on pourrait qualifier de Not In My Frontbeach (NIMF), à l'image de tel juge d'instruction originaire de Goianinha déposant plainte au tribunal contre le maire pour demander le retrait de toilettes publiques installées devant chez lui ${ }^{46}$ - mais le processus paraît inéluctable. Comme par un retour aux plantations, c'est désormais dans sa maison de campagne (la châcara) qu'Ormuz Simonetti se retire pour retrouver la quiétude et l'entre-soi des vacances familiales : "Loin du tumulte et des agglomérations. C'est le lieu parfait pour profiter de la famille et recycler nos vies, comme personnes et comme chrétiens ${ }^{47} »$.

\section{L'émergence \\ des élites autochtones}

La déprise de l'aristocratie locale est ensuite politique : elle se manifeste par la dissociation du pouvoir politique local vis-à-vis des fiefs sucriers et l'émergence de nouvelles élites parmi les populations autochtones. Ce rééquilibrage des rapports de forces politiques entre élites agraires et populations côtières s'amorce bien avant le développement touristique, avec les transformations du secteur primaire et les reconfigurations des frontières municipales. La modernisation de la pêche amorcée dans les années 1940 se traduit par des avancées technologiques importantes (motorisation, réfrigération, transport), une augmentation des rendements et un accroissement des inégalités entre pêcheurs. Le droit à la retraite est officiellement concédé en 1941 aux pêcheurs, dès lors reconnus comme «travailleurs de la mer », tandis que le gouvernement militaire encourage l'industrialisation du secteur en créant la Superintendance du développement de la pêche (1967) et encadre l'activité en installant des associations de pêcheurs, dites « colonies de pêche », le long du littoral. Le développement des bassins d'élevage de crevettes et de la pêche industrielle à la langouste à partir des années 1960 génère également d'importants revenus. L'arrivée de nouveaux acteurs économiques (armateurs, commerçants, industriels) et politiques 


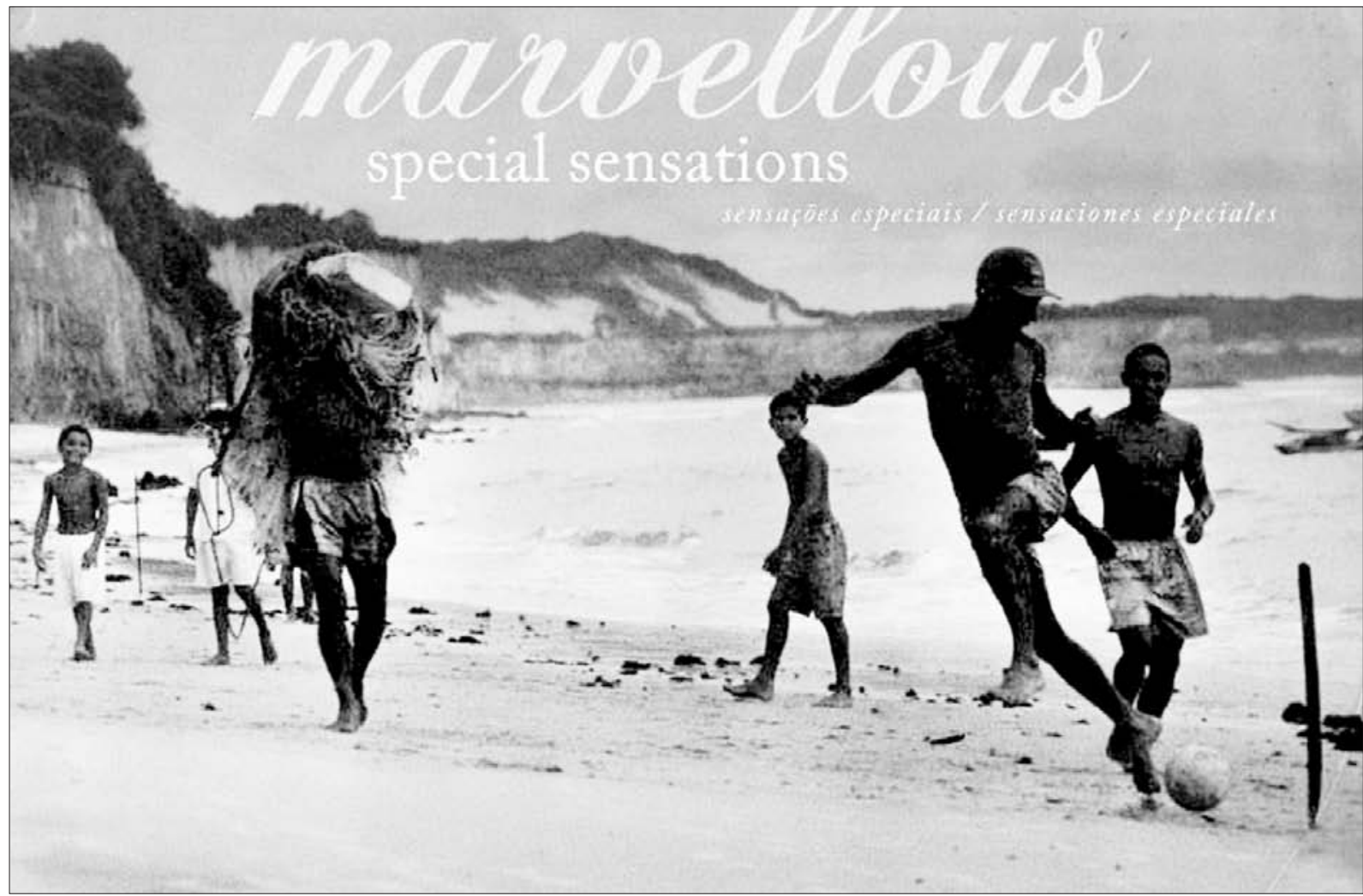

ILUSTRATION 11. La population locale devient en elle-même un motif d'attraction, comme sur cette couverture de magazine datant des années 1980.

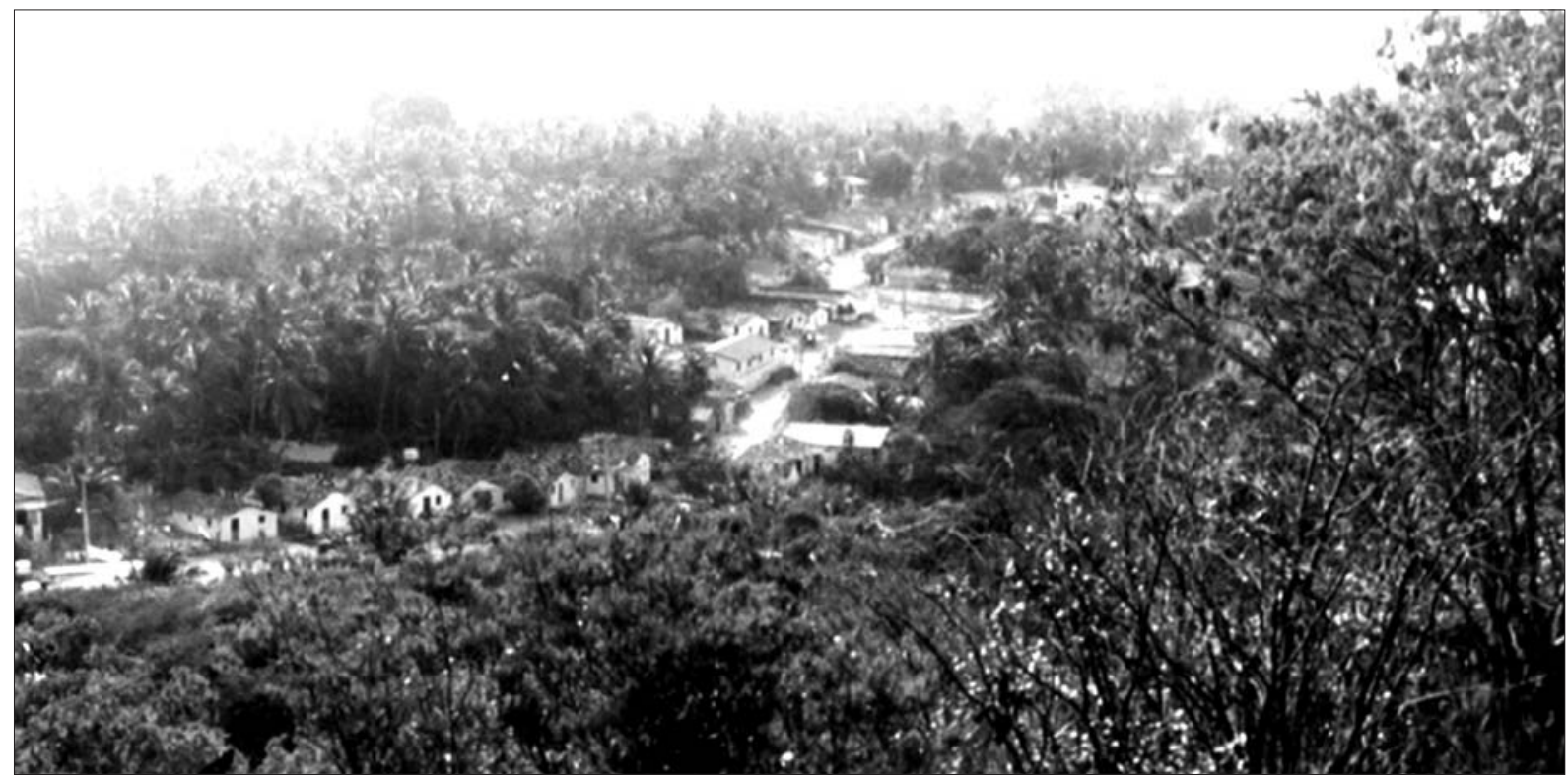

ILLUSTRATION 12. Le regard des nouveaux arrivants se tourne vers le centre du village. Ici une vue sur la rue principale de Pipa, perspective rare en comparaison avec les innombrables clichés de la plage. 


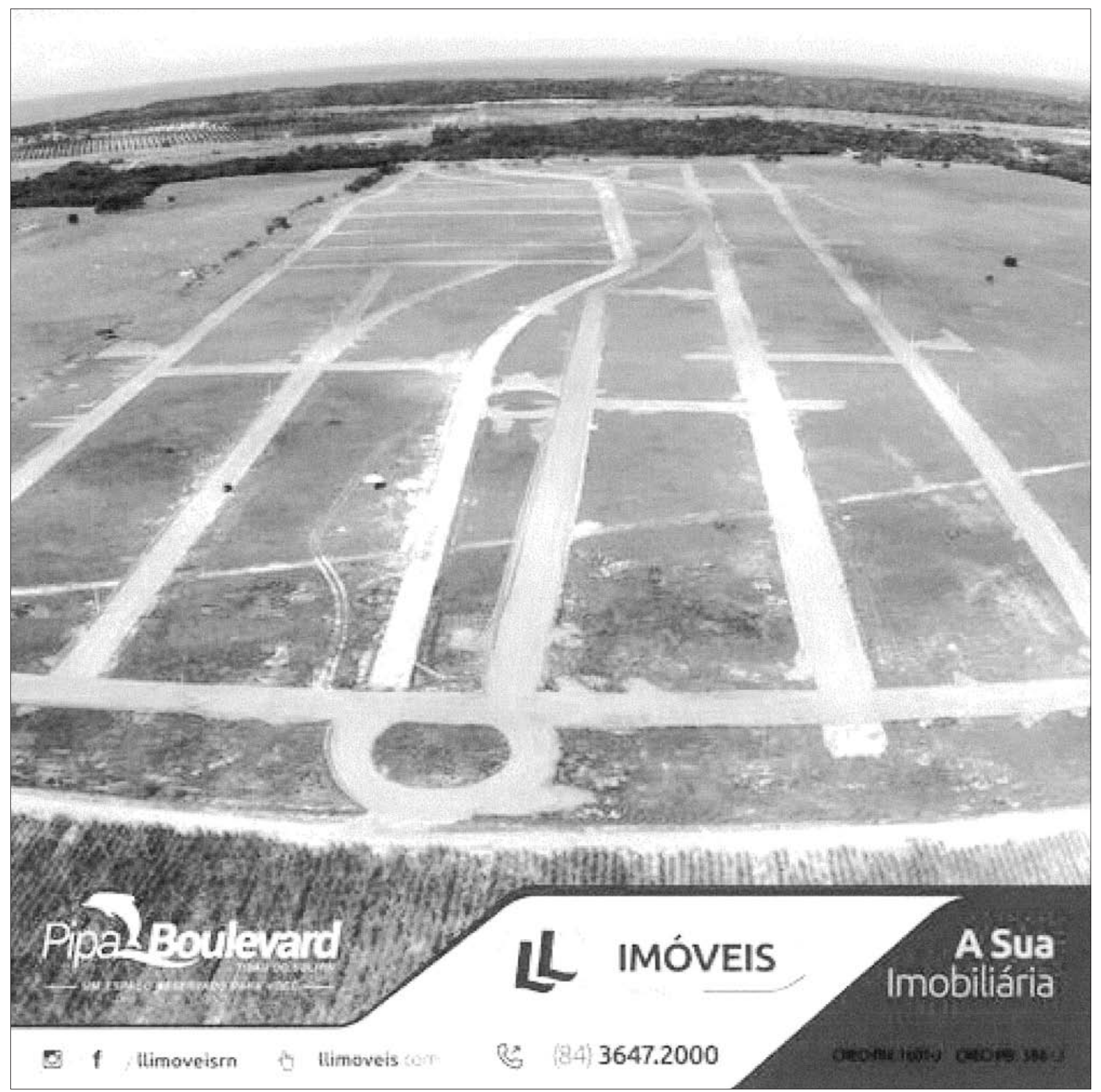

ILLUSTRATION 13. Lancement d'un grand lotissement à la périphérie de Pipa, en lieu et place d'un ancien champ de canne à sucre. 


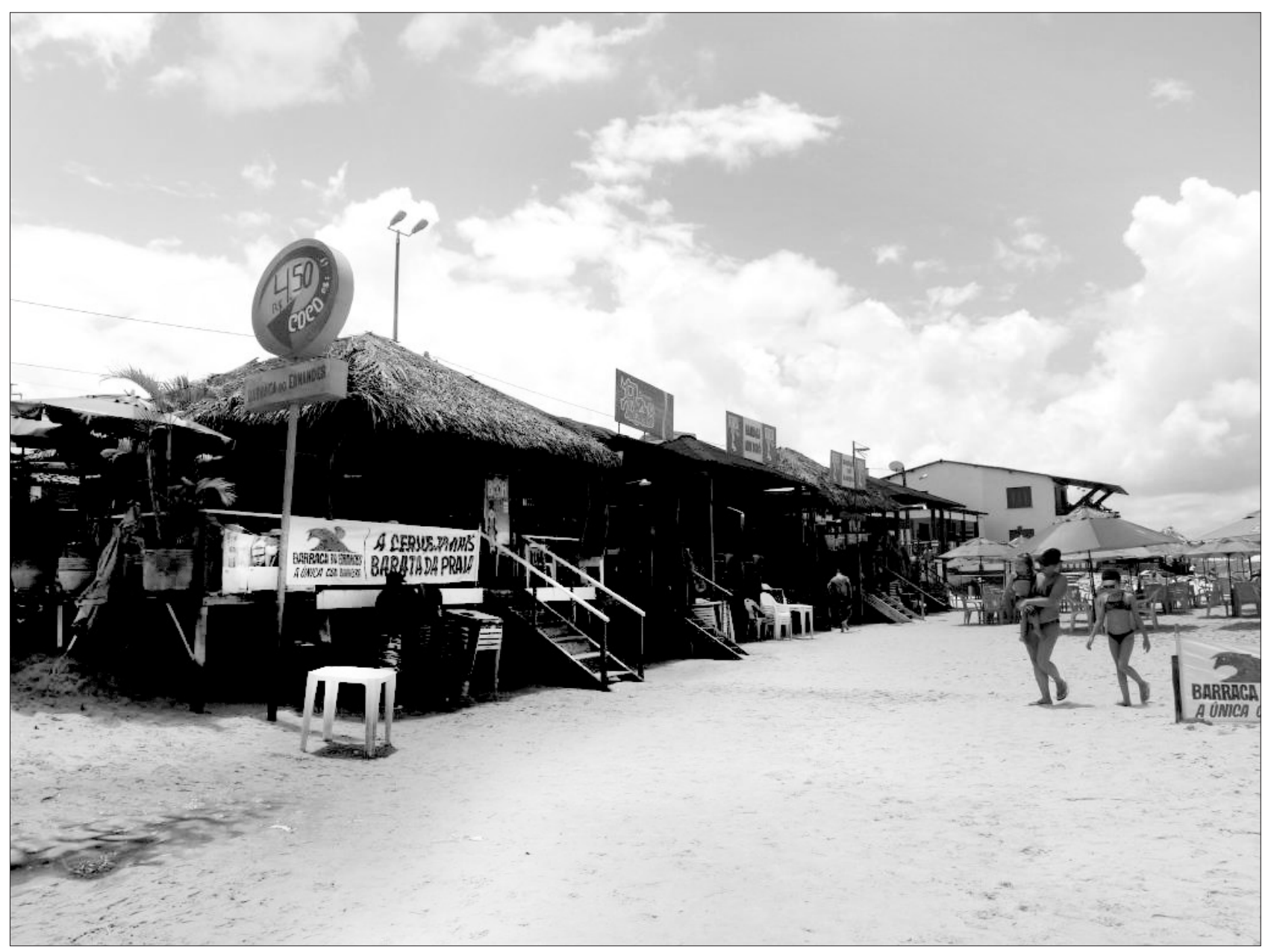

ILLUSTRATION 14. Les baraques s'interposent entre la plage et les maisons de villégiateurs. 
(syndicats, coopératives, administrations publiques), étrangers aux formes de solidarité communautaire, précipite le développement de ce que Conrad $\operatorname{Kottak}^{48}$ associe à une "éthique protestante » wébérienne fondée sur le mérite personnel et la capacité d'entreprendre, qui se substitue aux valeurs de savoir-faire et de solidarité communautaire des « bons capitaines». La réussite économique devient un attribut déterminant dans l'émergence de nouveaux leaders autochtones. Sur le plan agricole, la mécanisation, la généralisation des engrais chimiques et les subventions publiques contribuent à la revalorisation des terres côtières et à l'intensification des pressions foncières sur ces espaces autrefois délaissés. À Pipa, la fragmentation foncière et l'absence de régulations formelles des titres de propriété font obstruction à la modernisation et à l'expansion du grand capital agricole. Alors que le latifundium est contenu à la lointaine périphérie du village, le marché foncier local reste un pré carré économique pour une petite bourgeoisie foncière établie à Pipa, qui profite de ces nouvelles opportunités agricoles et bientôt de l'augmentation du prix des terrains touristiques.

Cette reconfiguration économique et sociale se traduit institutionnellement par l'émancipation municipale en 1963 de Tibau do Sul vis-à-vis de Goianinha, fief politique des élites sucrières. Cette autonomisation politique est provoquée par trois principaux facteurs : 1) le développement économique des territoires côtiers grâce à la modernisation de la pêche qui confère aux communautés côtières l'assise fiscale requise pour créer de nouvelles municipalités, 2) une conjoncture institutionnelle propice à la municipalisation, 3) le « portage » symbolique et politique d'un enfant du pays, Hélio Galvão ${ }^{49}$, un intellectuel amoureux des plages de Tibau et juriste influent parmi les cercles politiques opposés à l'oligarchie agraire (sucrière) qui va dessiner les frontières de la nouvelle commune en fonction des territoires affectifs de son enfance. La création de la commune de Tibau do Sul consolide la position des leaders locaux face aux élites régionales, basées à Goianinha. Cette reconfiguration des institutions municipales permet l'afflux de fonds fédéraux et facilite l'aménagement du territoire littoral (infrastructures de base), préalablement au développement touristique. Parmi la population locale, ceux qui parviennent à tirer profit du tourisme sont en général ceux qui parvenaient déjà à tirer leur épingle du jeu des transformations économiques à l'œuvre dans les secteurs maritime et agricole. On voit ainsi apparaître au sein des communautés du littoral de nouveaux leaders, réinvestissant dans le foncier ou dans le commerce les excédents de leur activité maritime (et réciproquement), et usant de leur connaissance du milieu pour conquérir le pouvoir politique - à l'image de José Odécio, agent de santé en charge de la prévention des épidémies (fièvre jaune, dengue), convertissant en capital électoral sa popularité acquise au cours des visites de routine dans les foyers de la commune, maire de 1982 à 1988 ; ou encore de Nivaldo Barros, commerçant et armateur, maire à deux reprises de 1976 à 1982 puis de 1988 à 1992.

L'emprise des leaders autochtones sur l'institution municipale persiste encore aujourd'hui en dépit de l'apparente diversification sociale de la commune. L'électorat autochtone reste en effet largement majoritaire et surtout soudé par des solidarités familiales, territoriales, religieuses (communautés évangélistes ou catholiques) ou professionnelles (syndicats de pêcheurs, baraquiers) ; alors que la population néo-résidente, démographiquement inférieure, apparaît politiquement atomisée et mal intégrée aux clientèles électorales locales. Le personnel politique municipal de Tibau do Sul est par conséquent composé essentiellement de membres d'anciennes familles locales descendantes de pêcheurs et d'agriculteurs. Pour ces familles, la politique est une ressource d'autant plus importante que le marché touristique offre des chances limitées de réussite sociale. Rares en effet sont les entrepreneurs autochtones parmi la classe hôtelière dominante, leur travail se limitant davantage à des métiers secondaires (dans le domaine des services ou de la construction) ou au petit commerce indépendant. Les administrations municipales constituent par conséquent une source d'emploi, à travers à la fois les fonctions électives et l'ensemble des postes dépendant directement ou indirectement des représentants au sein de la commune (chauffeur, agent d'entretien, employé de l'administration, etc.). En redistribuant les recettes fiscales sous forme de postes politiques (par cooptation) ou d'actions ciblées en direction des fractions autochtones (au gré des stratégies électoralistes), la politique municipale contribue à compenser les inégalités structurelles du marché touristique.

Contre le constat souvent simpliste d'une éviction des populations locales présentées comme cibles passives des impacts du tourisme, l'analyse sociohistorique du cas de Pipa met en évidence leur importance en tant que récepteurs actifs. Étudier la balnéarisation du littoral implique de questionner tout le spectre des producteurs, des avant-gardes ou prescripteurs extérieurs (médecins hygiénistes, artistes, surfeurs, voyageurs pionniers, etc.) aux acteurs endogènes $\mathrm{du}$ territoire. C'est en reconstruisant les trajectoires et positions de chacun de ces groupes, leurs antagonismes et leurs alliances tacites, leurs concessions et adaptations réciproques, que l'on comprend pourquoi et comment certains espaces ont pu être « touristifiés ».

À Pipa, l'arrivée des surfeurs et autres voyageurs bohèmes est sans doute déterminante pour comprendre l'identité touristique actuelle de la station, son orientation cosmopolite et festive, son ouverture sur l'international et en même temps la mise en valeur des codes culturels locaux. Mais il faut mettre cette $"$ intrusion balnéaire $»^{50}$ en regard avec la déprise relative de l'aristocratie sucrière sur ce territoire et le renforcement des positions de certaines fractions autochtones grâce aux transformations du secteur primaire (pêche, agriculture) et à l'émancipation municipale. Quelle aurait pu être la trajectoire de la destination si Pipa avait été un pré carré des puissants ? Si au lieu de paysans-pêcheurs aux revenus modestes mais indépendants, les surfeurs avaient trouvé une communauté de travailleurs ruraux serviles et désœuvrés comme il y en avait beaucoup ailleurs sur le littoral ? L'existence de bons spots de surf ne suffit pas à expliquer le succès du tourisme à Pipa, c'est sans 
doute aussi parce que le profil des habitants locaux - « simples mais fiers »- cadrait avec les valeurs "d'authenticité » attendues par les nouveaux arrivants et les touristes.

Une autre conclusion de ce travail revient à questionner les représentations de la plage comme espace autonome, la ligne de côte comme unité territoriale homogène. On ne peut ignorer dans l'analyse des configurations balnéaires l'influence des arrière-pays, des espaces portuaires, des métropoles voisines, de ce que Marcel Mauss appelait le « facteur tellurique ${ }^{51}$ » (les particularités topographiques et environnementales), ou encore des structures foncières qui sont la cristallisation territoriale d'anciens rapports sociaux de production. Le tropisme de la plage a progressivement focalisé le regard sur l'espace restreint de la grève de sable, confinant les profondeurs du territoire côtier au statut de coulisses de la scène touristique balnéaire, alors même que pour les populations locales ces espaces sont restés essentiels dans la perpétuation de la vie quotidienne, des imaginaires locaux, des systèmes politico-administratifs et des héritages sociaux. Derrière l'apparente légèreté des mœurs de plage se cachent bien souvent les pesanteurs historiques de plantations oubliées. 